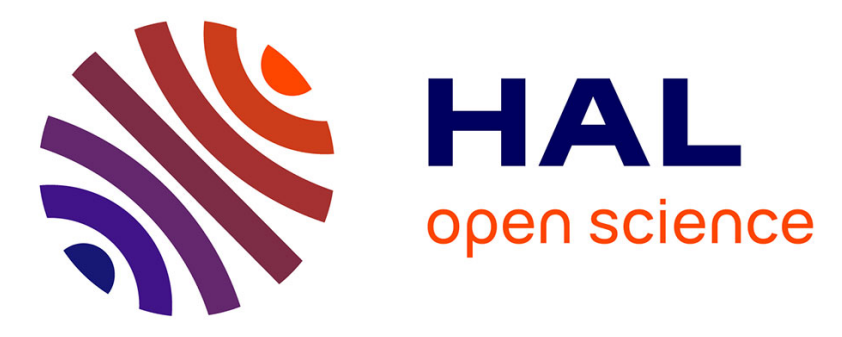

\title{
Interaction of Cationic, Anionic, and Nonionic Macroraft Homo- and Copolymers with Laponite Clay
}

Thaíssa de Camargo Chaparro, Rodrigo Duarte Silva, Igor Stefanichen Monteiro, A. Barros-Timmons, Reinaldo Giudici, Amilton Martins dos Santos, Elodie Bourgeat-Lami

\section{To cite this version:}

Thaíssa de Camargo Chaparro, Rodrigo Duarte Silva, Igor Stefanichen Monteiro, A. Barros-Timmons, Reinaldo Giudici, et al.. Interaction of Cationic, Anionic, and Nonionic Macroraft Homo- and Copolymers with Laponite Clay. Langmuir, 2019, 35 (35), pp.11512-11523. 10.1021/acs.langmuir.9b01987 . hal-02325049

\section{HAL Id: hal-02325049 \\ https://hal.science/hal-02325049}

Submitted on 12 Nov 2020

HAL is a multi-disciplinary open access archive for the deposit and dissemination of scientific research documents, whether they are published or not. The documents may come from teaching and research institutions in France or abroad, or from public or private research centers.
L'archive ouverte pluridisciplinaire HAL, est destinée au dépôt et à la diffusion de documents scientifiques de niveau recherche, publiés ou non, émanant des établissements d'enseignement et de recherche français ou étrangers, des laboratoires publics ou privés. 


\title{
Interaction of cationic, anionic and non-ionic macroRAFT homo and copolymers with Laponite clay
}

\author{
Thaíssa de Camargo Chaparro, ${ }^{\mathrm{a}, \mathrm{b}}$ Rodrigo Duarte Silva, ${ }^{\mathrm{b}}$ Igor Stefanichen Monteiro, ${ }^{\mathrm{b}}$ A. Barros- \\ Timmons, ${ }^{\mathrm{c}}$ Reinaldo Giudici, ${ }^{\mathrm{d}}$ Amilton Martins dos Santos, ${ }^{\mathrm{b}^{*}}$ Elodie Bourgeat-Lami ${ }^{{ }^{*}}$
}

\begin{abstract}
${ }^{a}$ Univ Lyon, Université Claude Bernard Lyon 1, CPE Lyon, CNRS, UMR 5265, Chemistry, Catalysis, Polymers and Processes (C2P2), 43, Bvd du 11 Novembre 1918, F-69616 Villeurbanne, France. ${ }^{b}$ Engineering School of Lorena - University of São Paulo, 12.602-810 Lorena/SP, Brazil. ' Department of Chemistry, CICECO, University of Aveiro, Campus Universitário de Santiago, 3810-193 Aveiro, Portugal. ${ }^{d}$ Department of Chemical Engineering, Polytechnic School of the University of São Paulo, 05508-010 São Paulo/SP, Brazil.
\end{abstract}

\begin{abstract}
The functionalization of Laponite ${ }^{\circledR}$ RD platelets with different cationic, anionic and non-ionic homo and copolymers synthesized by reversible addition-fragmentation chain transfer (RAFT) has been investigated. The effective interaction of the macromolecular RAFT agents (macroRAFT) with the inorganic particles is known to be of crucial importance for the successful coating of minerals with polymers via RAFT-mediated emulsion polymerization to produce polymer-encapsulated inorganic particles. The macroRAFT agents synthesized in the present work contain carefully selected re-initiating $\mathrm{R}$ groups, which bear either ionizable tertiary amine or quaternary ammonium moieties (from 2-(dimethylamino)ethyl methacrylate, DMAEMA), negatively charged acrylic acid (AA) repeat units or neutral polyethylene glycol (PEG) side chains, and are capable of interacting with Laponite via different adsorption mechanisms. The equilibrium adsorption of these RAFT (co)polymers was investigated by the plotting of adsorption isotherms, and either L-type or H-type curves were obtained. The hydrophobicity of the macroRAFT was shown to promote adsorption, as did the pending configuration of the PEG block. Charge repulsion between AA and the negatively charged surface of Laponite at $\mathrm{pH} 7.5$, on the other hand, was prejudicial for adsorption, while the strong electrostatic interaction between the cationic DMAEMA molecules and the Laponite surface led to high-affinity-type curves.
\end{abstract}




\section{Introduction}

Organic/inorganic nanocomposite latexes ${ }^{1}$ represent an innovative branch in materials science that has attracted considerable attention in recent years owing to the various applications of the resulting products, from the coating to the pharmaceutical industries for instance. The incorporation of inorganic nanoparticles into polymeric matrixes associates the best advantages of inorganics with the processability and flexibility of polymers, bringing significant improvements to the final properties of the material, as compared to the corresponding pure polymers. ${ }^{2,3}$ When the polymeric matrix and the inorganic part interact with good affinity, the fillers can be homogeneously distributed in the matrix, and the overall properties can be significantly improved. However, these components do not present, typically, a good affinity with each other, so special attention must be paid to this matter to avoid phase separation. One of the most currently used strategies to increase the affinity between organic and inorganic parts is the modification of the inorganic particles with organic molecules, such as small ligands or longer polymeric chains. In the case of nanocomposites prepared by emulsion polymerization, controlling the phase separation, and hence the resulting particle morphology, is even more challenging due to the complexity of this nonequilibrium multiphase reaction process which involves a multitude of chemical and physical phenomena.

The use of reversible deactivation radical polymerization (RDRP) has been an attractive strategy to overcome the difficulties found in the synthesis of organic/inorganic nanocomposites by emulsion polymerization. ${ }^{4}$ In short, the approach relies on the use of hydrophilic living copolymers that work as coupling agents and stabilizers to generate hybrid latex particles with controlled morphologies. $5,4,6$ 
Although there has been a few reports on nitroxide-mediated polymerization ${ }^{7,} 8,9,10$ and atom transfer radical polymerization, ${ }^{11}$ most works in the field have relied on the use of reversible addition-fragmentation chain transfer (RAFT) polymerization. RAFT is a versatile technique for the controlled synthesis of various polymer architectures that can operate under mild temperatures, is suitable for a broad variety of monomers and can be performed in a wide range of solvents. All these advantages make the RAFT process particularly well suited to produce organic/inorganic nanocomposite latex particles. In 2008, the strategy was first described by Nguyen et al. ${ }^{12}$ and Daigle et al. ${ }^{13}$ to encapsulate different inorganic particles. The method developed, which was recently called macroRAFT-assisted encapsulating emulsion polymerization (REEP), ${ }^{6}$ is based on the use of amphiphilic macroRAFT agents to promote the interaction between the inorganic surface and the hydrophobic polymeric block, which is formed in situ by the chain extension of the macroRAFT, generating the encapsulating shell around the inorganic particle. For this purpose, hydrosoluble control agents must be initially adsorbed onto the inorganic particles, and then submitted to a chain extension with hydrophobic monomers in aqueous media, generating amphipathic block copolymers either directly on the surface of the particles or in the aqueous phase, where they suffer a self-assembly process. Diverse particles have been successfully encapsulated by the REEP technique, including cadmium sulfide ${ }^{14}$ and lead sulfide ${ }^{15}$ quantum dots, cerium oxide, ${ }^{16}$ silica $^{17}$ iron oxide, ${ }^{18,}, 19$ carbon nanotubes, ${ }^{20,} \quad 21$ Gibbsite, ${ }^{22}$ layered double hydroxides (LDH), ${ }^{23,24,25}$ Montmorillonite clay (MMT) ${ }^{26,27}$ and graphene oxide. ${ }^{28}$

The interaction between the macroRAFT agent and the inorganic surface was shown to be of key importance for the encapsulation of inorganic particles through the REEP strategy. The RAFT copolymers allow the establishment of an effective physical link 
between the inorganic particle and the polymer shell and, with the displacement of the polymerization locus from the aqueous medium to the inorganic surface, selfstabilizing hybrid particles can be generated in situ. A strong adsorption of the macromolecules onto the particle, with a good coverage of its surface and minimal amount of free molecules in the aqueous phase, guarantees that the macroRAFTs can act as effective coupling agents and precursors of stabilizers for the nanocomposites.

Following this direction and seeking a better understanding of the relationship between the adsorption of macroRAFT agents and the encapsulation process by REEP, the aim of the present work is to study the surface modification of Laponite ${ }^{\circledR} \mathrm{RD}$, a synthetic clay, with different macroRAFT agents containing anionic, cationic or non-ionic moieties and evaluate, by means of experimental adsorption isotherms, how the structure of the macroRAFT agents affects their interaction with the clay surface. The obtained results are compared with previously reported data on MMT clay and discussed considering the differences in the physico-chemical properties between the two clay minerals.

Regarding the design of the molecules, the $\mathrm{Z}$ and $\mathrm{R}$ groups of the RAFT agent were carefully selected. The 4-cyano-4-thiothiopropylsulfanyl pentanoic acid (CTPPA), a (thiocarbonyl)sulfanyil chain transfer agent that carries a short hydrophobic alkyl chain end (thiopropyl) as $\mathrm{Z}$ group, was chosen as RAFT agent. To allow strong interaction with the inorganic particles, a series of different macroRAFTs with the $\mathrm{R}$ group bearing either an ammonium (or quaternized ammonium) group (from $\mathrm{N}, \mathrm{N}-$ dimethylaminoethyl methacrylate, DMAEMA), anionic groups (i.e., acrylic acid: AA) or neutral polyethylene glycol (PEG) side chains, were synthesized by solution polymerization in the presence of CTPPA. 
The experimental data obtained from the equilibrium adsorption isotherms were fitted to various models and the characteristic adsorption parameters obtained were used to provide insights into the adsorption mechanisms of each macroRAFT on Laponite in aqueous medium.

\section{Experimental}

Materials. 4-4'-Azobis-4-cyanovaleric acid (ACPA, $\geq$ 98\%, Sigma-Aldrich), 1,3,5-trioxane (>99\%, Sigma-Aldrich) and $n$-hexane (95\% Vetec) were all used as received. 1,4-Dioxane (>99.5\%), diethyl ether (>99.5\%) and petroleum ether (p. a.) were purchased from SigmaAldrich and used without further purification. Trimethylsilyldiazomethane $\left(\mathrm{TMSCHN}_{2}, 2 \mathrm{M}\right.$ solution in diethyl ether, Aldrich) was used for the methylation of carboxyl groups of AAcontaining macroRAFT agents and iodomethane $\left(\mathrm{ICH}_{3}\right.$, Vetec, 99\%) was used for the quaternization of DMAEMA-based macroRAFTs. Both reagents were used as received. Poly(ethylene glycol) methyl ether (mPEG, $2000 \mathrm{~g} \mathrm{~mol}^{-1}$, Fluka, 99.5\%) was distilled twice with toluene before use. The monomers: acrylic acid (AA, anhydrous, 99\% Sigma-Aldrich), poly(ethylene glycol) methyl ether acrylate (PEGA, $M_{\mathrm{n}}=480 \mathrm{~g} \mathrm{~mol}^{-1}, 99 \%$, Sigma-Aldrich), 2-(dimethylamino)ethyl methacrylate (DMAEMA, 99\% stabilized, Acros Organics) and $n$ butyl acrylate (BA, 99\%, Sigma-Aldrich) were all used as received. Deuterated chloroform $\left(\mathrm{CDCl}_{3}, 100 \%\right.$, Aldrich), deuterium oxide $\left(\mathrm{D}_{2} \mathrm{O}\right)$ and dimethyl sulfoxide- $\mathrm{d}_{6}\left(\right.$ DMSO- $_{6}, 99 \%$, Sigma-Aldrich) were used as solvents for proton nuclear magnetic resonance analysis $\left({ }^{1} \mathrm{H}\right.$ NMR). Tetrahydrofuran (THF, 99.9\%, HPLC grade, Aldrich) was filtered and degassed in an ultrasound bath before being used as solvent in size exclusion chromatography (SEC) analysis. Triethylamine (TEA, 99\%, Vetec) was used as additive in THF for SEC analysis of DMAEMA-based macroRAFT agents. The water used in the adsorption experiments was deionized (Purelab Classic UV, Elga LabWater). 
Laponite $^{\circledR}$ RD. The clay mineral chosen for this work was Laponite ${ }^{\circledR}$ RD, a synthetic hectorite produced by BYK Additives Ltd (former Rockwood Additives Ltd, UK). Laponite has a layer structure comparable with those of natural hectorite and bentonite, being disposed in the form of disc-shaped crystals with diameter of $\sim 30 \mathrm{~nm}$ and thickness of $\sim 0.92 \mathrm{~nm}$, when dispersed in water. In the smectite group, hectorite and MMT are among the most frequently used clays for the preparation of nanocomposites. ${ }^{29,30,31,32,33}$ These materials have unique properties, such as high ionic exchange capacity, large chemically active surface area and special hydration characteristics. In the idealized structure of Laponite, the central octahedral sheet contains six divalent magnesium ions (giving a positive charge of twelve) and is sandwiched between two tetrahedral layers containing four silicon atoms. Twenty atoms of oxygen and four hydroxyl groups complete the balance of these layers, forming the magnesia octahedral and the silica tetrahedral sheets, which should have a neutral charge. However, in practice, Laponite has a negative surface charge that can be attributed to two factors: the isomorphic substitution of magnesium ions within the lattice structure by monovalent lithium ions and the broken edges. ${ }^{34}$ The negative charges of the crystal faces are counterbalanced by the adsorption of cations (predominantly $\mathrm{Na}^{+}$) in the interlayer space of Laponite, corresponding to a cation exchange capacity (CEC) of $0.75 \mathrm{meq} \mathrm{g}^{-1}$. It has been reported that

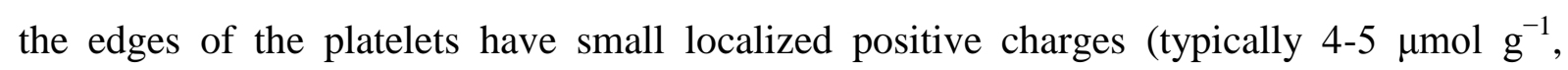
representing less than $10 \%$ of the CEC), that slightly decrease with increasing the $\mathrm{pH}^{35}$ The edge of the crystal structure contains predominantly $\mathrm{MgOH}$ amphoteric groups that can be protonated or deprotonated depending on the $\mathrm{pH}$ of the medium. Above the $\mathrm{pH}$ associated with point of zero charge (PZC) of the edges, which has been indicated to be 11 in the case of Laponite particles, ${ }^{36} \mathrm{H}^{+}$ions dissociate from the edges (rendering them negative) while, below the $\mathrm{PZC}, \mathrm{OH}^{-}$ions are released (and the edges become positively charged. The $\mathrm{pH}$ has a strong influence on the stability of the Laponite dispersion. Laponite dispersions are stable 
predominantly at high $\mathrm{pH}$, being found at $\mathrm{pH}$ around $10 .{ }^{37}$ Under acidic conditions, the leaching of $\mathrm{Mg}^{2+}$ ions takes place and the increase in the ionic strength of the medium and on the positive charges of the edges favor rim-to-face interactions, increasing the gelation rate. MMT platelets, in contrast, present a PZC of 6.5 and, therefore, the neutralization of edges charges takes place under more acidic conditions (reducing the range of $\mathrm{pH}$ for which the platelets can undergo edge-to-face interaction). ${ }^{38}$ In addition, Laponite and natural sodium MMT can also be differentiated in some other important aspects, such as the much larger aspect ratio and less uniform dispersity of MMT platelets, together with the different structural organization of their octahedral layers and their different CEC values $\left(0.92 \mathrm{meq} \mathrm{g}^{-1}\right.$ for MMT and $0.75 \mathrm{meq} \mathrm{g}^{-1}$, for Laponite). ${ }^{39}$ Moreover, Laponite has some advantages, when compared for instance to MMT, that make it an ideal model substrate, such as a high chemical purity, a uniform dispersity of the elementary platelets and the ability to produce clear dispersions. The anisotropic shape of the platelets and their thermal and mechanical properties make Laponite an interesting material to be used as inorganic filler. Among the different grades of Laponite available, the RD grade is the most frequently studied. It is a fastdispersing gel-forming grade that does not contain any added peptizer. Peptizer tetrasodium pyrophosphate $\left(\mathrm{Na}_{4} \mathrm{P}_{2} \mathrm{O}_{7}, 95 \%\right.$, Aldrich) was added to Laponite powder for adsorption studies carried out with non-ionic macroRAFT agents (in which neither the edges nor the surfaces of Laponite were covered with ionizable macroRAFT agents) to hinder gel formation, as this tetravalent negatively charged ion adsorbs on the rims of the platelets and protects them from rim-to-face interactions and the formation of "house of cards" structures. ${ }^{40,41}$ Laponite ${ }^{\circledR} \mathrm{RD}$ (without added peptizer) was used in all other cases in order to also allow interaction with the clay edges.

Synthesis of RAFT and macroRAFT agents. The RAFT agent: 4-cyano-4-thiothio propyl sulfanyl pentanoic acid (CTPPA) was obtained as described elsewhere. ${ }^{17}$ Different 
trithiocarbonate macroRAFT agents were synthesized from CTPPA. For the synthesis of MR1, MR2 and MR11 macroRAFT agents by solution polymerization (see Table 1), initially, the chain transfer agent CTPPA was added to a two-necked round-bottom flask equipped with a condenser, where the initiator, ACPA (10\% of the molar concentration of RAFT agent) and the monomers were added. 1,3,5-Trioxane was used as an internal reference for determination of monomer conversion by ${ }^{1} \mathrm{H}$ NMR, in a molar ratio of $1 / 12$ related to monomer(s). The mixture was dissolved in a proper amount of 1,4-dioxane, so the final concentration of macroRAFT agent in the solvent would not exceed $25 \mathrm{wt} \%$, and the reaction medium was purged for $30 \mathrm{~min}$ with nitrogen and then heated to $80^{\circ} \mathrm{C}$ to start polymerization. Samples were taken periodically, and after $\sim 7-8$ hours, the polymerization was quenched by immersing the flask into an ice bath. The final products were submitted to 3 precipitation cycles using a large volume of cold $n$-hexane or petroleum ether and dried overnight under vacuum at room temperature. The PEG-CTPPA macroRAFT agent (MR3, Table 1), was synthesized via an esterification reaction between a $2000 \mathrm{~g} \mathrm{~mol}^{-1} \mathrm{mPEG}$ and CTPPA, according to the procedure described in the literature. ${ }^{16}$ Some of the homo and copolymers synthesized were used, after purification, as a precursor in chain extension reactions, to obtain block copolymers (MR4, MR5 and MR7, Table 1). The synthesis of these macroRAFT copolymers was carried out using a similar procedure to the one previously described, but the chain transfer agent CTPPA was replaced, in these cases, by the macroRAFT agents: PEG-CTPPA (MR3) and PAACTPPA (MR1). The macroRAFT agents $\mathrm{P}\left(\mathrm{PEGA}_{6}-c o-\mathrm{BA}_{4}\right)-\mathrm{CTPPA}(\mathrm{MR} 6)^{17} \mathrm{P}\left(\mathrm{AA}_{4}\right.$-co$\left.\mathrm{PEGA}_{4}-\mathrm{co}-\mathrm{BA}_{4}\right)-\mathrm{CTPPA} \quad(\mathrm{MR} 8), \quad \mathrm{P}\left(\mathrm{AA}_{9}-c o-\mathrm{PEGA}_{9}-c o-\mathrm{BA}_{9}\right)-\mathrm{CTPPA} \quad(\mathrm{MR} 9) \quad$ and P(DMAEMA $\left.10^{-}-c o-B_{5}\right)$-CTPPA (MR10) were synthesized as described elsewhere. ${ }^{42}$ MR10 and MR11 were submitted to a quaternization reaction with $\mathrm{ICH}_{3}$ in $\mathrm{THF}$ for $24 \mathrm{~h}$, according to the literature procedure. ${ }^{43,44}$ The products were filtered and dried in vacuum oven to 
remove the excess of solvent and $\mathrm{ICH}_{3}$. The amounts of chain transfer agent and monomers used in the synthesis are shown in Table 1.

Proton nuclear magnetic resonance spectroscopy $\left({ }^{1} \mathbf{H}\right.$ NMR). For the synthesis of macroRAFT agents, the final monomer conversions were monitored by ${ }^{1} \mathrm{H}$ NMR at room temperature (Bruker DRX 300), by diluting the crude reaction medium in DMSO- $\mathrm{d}_{6}$. 
Table 1 MacroRAFT agents synthesized and recipes used in the synthesis ([ACPA]/[CTA] = 1/10 mol/mol); overall monomer conversion, theoretical and experimental molar mass and dispersity of all macroRAFT agents synthesized in this work.

\begin{tabular}{|c|c|c|c|c|c|c|c|c|c|c|}
\hline \multirow[t]{2}{*}{ Entry } & \multirow[t]{2}{*}{ MacroRAFT $^{\mathrm{a}}$} & \multirow{2}{*}{$\frac{[\mathrm{CTA}]}{(\mathbf{m M})}$} & \multicolumn{4}{|c|}{$[\text { Monomer }]_{0}(\mathbf{M})$} & \multirow{2}{*}{$\frac{X^{\mathbf{b}}}{(\%)}$} & \multirow{2}{*}{$\frac{M_{\mathrm{n}, \text { theo }}}{\left(\mathrm{g} \mathrm{mol}^{-1}\right)}$} & \multirow{2}{*}{$\frac{M_{\mathrm{n}, \exp }}{\left(\mathrm{g} \mathrm{mol}^{-1}\right)}$} & \multirow[t]{2}{*}{$\boldsymbol{\oplus}$} \\
\hline & & & $\mathbf{A A}$ & PEGA & BA & DMAEMA & & & & \\
\hline MR1 & $\mathrm{PAA}_{40}$-CTPPA & 60.0 & 2.50 & - & - & - & 95 & 3120 & 3630 & 1.19 \\
\hline $\mathrm{MR}^{\mathrm{c}}$ & $\mathrm{PEG}_{45}-\mathrm{CTPPA}$ & - & - & - & - & - & - & 2270 & 3590 & 1.09 \\
\hline$M R 4^{d}$ & $\mathrm{PEG}_{45}-b-\mathrm{PAA}_{42}-\mathrm{CTPPA}$ & 62.2 & 3.05 & - & - & - & 86 & 5310 & 6710 & 1.18 \\
\hline $\mathrm{MR}^{\mathrm{e}}$ & $\mathrm{PAA}_{40}-b-\mathrm{P}\left(\mathrm{PEGA}_{4}\right)-\mathrm{CTPPA}$ & 74.6 & - & 0.36 & - & - & 84 & 5460 & 4640 & 1.31 \\
\hline MR7 & $\mathrm{PAA}_{40}-b-\mathrm{P}\left(\mathrm{PEGA}_{6}-c o-\mathrm{BA}_{4}\right)-\mathrm{CTPPA}$ & 54.8 & - & 0.41 & 0.23 & - & 87 & 7320 & 6130 & 1.21 \\
\hline MR8 & $\mathrm{P}\left(\mathrm{AA}_{4}-c o-\mathrm{PEGA}_{4}-c o-\mathrm{BA}_{4}\right)-\mathrm{CTPPA}$ & 60.9 & 0.31 & 0.30 & 0.30 & - & 87 & 3100 & 3120 & 1.20 \\
\hline MR9 & $\mathrm{P}\left(\mathrm{AA}_{9}-c o-\mathrm{PEGA}_{9}-c o-\mathrm{BA}_{9}\right)-\mathrm{CTPPA}$ & 29.3 & 0.31 & 0.29 & 0.29 & - & 83 & 6230 & 5730 & 1.20 \\
\hline MR10 & $\mathrm{P}\left(\mathrm{DMAEMA}_{10}-c o-\mathrm{BA}_{4}\right)-\mathrm{CTPPA}$ & 91.4 & - & - & 0.46 & 0.90 & 87 & 2310 & 1630 & 1.42 \\
\hline MR11 & $\mathrm{P}\left(\mathrm{DMAEMA}_{19}-\mathrm{co}-\mathrm{BA}_{14}\right)$-CTPPA & 34.9 & - & & 0.68 & 0.68 & 81 & 4400 & 4520 & 1.45 \\
\hline
\end{tabular}

${ }^{\mathrm{a}}$ The actual number of repeat units in the polymer chain is based on the individual conversion of each comonomer. ${ }^{\mathrm{b}} X=$ overall monomer conversion; ${ }^{\mathrm{c}}$ Synthesized via esterification reaction between $\mathrm{mPEG}$ and CTPPA. ${ }^{\mathrm{d}}$ Synthesized using MR3, PEG-CTPPA, as precursor. ${ }^{\mathrm{e}}$ Synthesized using MR1, PAA-CTPPA, as precursor. 
By relying on the use of 1,3,5-trioxane as internal standard, the relative integration of the vinylic protons of the monomers led to the determination of monomer conversion. The final products obtained after the synthesis of RAFT agent CTPPA and macroRAFT agents, were dried under vacuum overnight at room temperature and characterized by ${ }^{1} \mathrm{H}$ NMR spectroscopy, using $\mathrm{CDCl}_{3}$ as solvent.

Size Exclusion Chromatography (SEC). The average molar masses (number-average molar mass, $M_{\mathrm{n}}$, and weight-average molar mass, $M_{\mathrm{w}}$ ) and the molar mass dispersity $\left(Ð=M_{\mathrm{w}} / M_{\mathrm{n}}\right)$ of the macroRAFT agents were determined by SEC. All samples were filtrated through a $0.45 \mu \mathrm{m}$ pore-size membrane and analyzed at the concentration of 5 $\mathrm{mg} \mathrm{mL}^{-1}$. The AA-based macroRAFT agents had their carboxylic acid groups methylated with $\mathrm{TMSCHN}_{2}$, in a $\mathrm{THF} / \mathrm{H}_{2} \mathrm{O}$ mixture before injection. ${ }^{45}$ The analyses were performed using a Viscotek TDA305 system from Malvern Instruments equipped with a differential refractive index $(\mathrm{RI})(\lambda=670 \mathrm{~nm})$ and static light scattering detectors (RALS, $90^{\circ}$, and LALS, $7^{\circ}$ ) and as well as a viscometer. The separation was performed at $40^{\circ} \mathrm{C}$ with $\mathrm{THF}$ as eluent at a flow rate of $1 \mathrm{~mL} \mathrm{~min}^{-1}$ on a set of columns that comprise a pre-column (PLgel Olexis Guard $7.5 \times 50 \mathrm{~mm}$ ) followed by three columns connected in series (PLgel Olexis Guard 7.5x300 mm). For the analysis of the DMAEMA-based macroRAFT agents, samples were injected in a Shimadzu HPLC equipment equipped with an automatic Sil-20A Shimadzu injector and a differential refractive index detector RID-A (Shimadzu). The eluent was THF with 0.3 wt\% of TEA at a flow rate of $0.8 \mathrm{~mL} \mathrm{~min}^{-1}$. The separation was carried out in two Phenogel columns $\left(5 \mu \mathrm{m}, 300 \times 7.8 \mathrm{~mm}^{2}\right)($ Phenomenex $)$ of $10^{4} \AA$ and $10^{6} \AA$ at $35^{\circ} \mathrm{C}$. The number and weight average molar masses $\left(M_{\mathrm{n}}, M_{\mathrm{w}}\right)$ and the dispersity $\left(\bigoplus=M_{\mathrm{w}} / M_{\mathrm{n}}\right)$ were obtained by deriving the RI signal and using a calibration curve based on poly(methyl methacrylate) (PMMA) standards from Polymer Laboratories. 
Adsorption of macroRAFTs onto Laponite. The interaction of AA-, PEG- and DMAEMA-based macroRAFT agents with Laponite was investigated and equilibrium adsorption isotherms were determined for each macroRAFT agent synthesized. For this purpose, a stock dispersion of Laponite was initially prepared by adding $0.4 \mathrm{~g}$ of Laponite into $20 \mathrm{~mL}$ of water and left for 30 minutes under magnetic stirring. Exclusively for the $\mathrm{PEG}_{45}-\mathrm{CTPPA}$ and $\mathrm{P}\left(\mathrm{PEGA}_{6}-\mathrm{co}-\mathrm{BA}_{4}\right)-\mathrm{CTPPA}$ adsorption isotherms, the peptizer was added to the Laponite powder in controlled amounts (10 wt $\%$ based on clay). Aliquots of $1 \mathrm{~mL}$ of the $20 \mathrm{~g} \mathrm{~L}^{-1}$ clay suspension were added to small flasks, where different volumes of a previously prepared macroRAFT agent solution were added. All flasks were completed with water until a final volume of 4 mL, obtaining macroRAFT/Laponite dispersions with a fixed final clay concentration of $5 \mathrm{~g} \mathrm{~L}^{-1}$ and different macroRAFT agent concentrations. The $\mathrm{pH}$ of each dispersion was carefully adjusted at this point, by adding droplets of $\mathrm{NaOH}$ or $\mathrm{HCl}$ aqueous solutions. The dispersion was stirred for approximately 15 hours to ensure that the adsorption had reached equilibrium as many works report that polymer adsorption on solid surfaces is fast, ${ }^{46}$ and further ultracentrifuged at $60000 \mathrm{rpm}$ for one hour, using a Sorvall ${ }^{\text {TM }}$ MTX 150 Micro-Ultracentrifuge, from Thermo Scientific. The supernatant was recovered for determination of the amount of free macroRAFT agent (the equilibrium concentration of macroRAFT agent in the supernatant, $C_{\mathrm{e}}, \mathrm{g} \mathrm{L}^{-1}$ ) by spectrophotometry, using a spectrophotometer Genesys ${ }^{\mathrm{TM}}$ 10UV from Thermo Scientific and optical glass cuvettes with $10 \mathrm{~mm}$ optical path, at a wavelength $(\lambda)$ of $310 \mathrm{~nm}$. The macroRAFT agents, which derive from CTPPA, contain a trithiocarbonate chain end that guarantees their absorbance in the ultraviolet-visible spectral region, presenting an intense absorption at $310 \mathrm{~nm} \cdot{ }^{47}$ A calibration curve of absorbance as a function of macroRAFT agent concentration was pre-determined at 
this wavelength by successive dilutions of a concentrated solution of macroRAFT agent. With the difference between the initial concentration of macroRAFT agent, $C_{0}$ ( $\mathrm{g} \mathrm{L}^{-1}$ ) and the equilibrium concentration, it was possible to calculate the adsorbed amount of macroRAFT agent, $q_{\mathrm{e}}\left(\mathrm{mg} \mathrm{g}^{-1}\right)$, according to:

$$
q_{e}=\frac{\left(C_{0}-C_{e}\right)}{C_{c l a y}} \times 1000
$$

where $C_{\text {clay }}\left(\mathrm{g} \mathrm{L}^{-1}\right)$ is the concentration of Laponite in the sample.

Some of the adsorption isotherms were randomly selected for duplicates, in order to verify the repeatability of the measurements, and no significant variation was observed in these cases.

Dynamic Light Scattering (DLS). The hydrodynamic particle diameter $\left(\mathrm{Z}_{\mathrm{av}}\right)$ and the particle size distribution (indicated by the PdI value; the higher this value, the broader the size distribution) were determined by DLS in a NanoZetasizer instrument from Malvern. Typically, one drop of the sample was diluted in pure deionized water before analysis and the reported particle size represents an average of 3 measurements. Even though this technique is more indicated for spherical particles, not providing an exact measurement of $Z_{\mathrm{av}}$. of platelets, it can be considered a useful indicative for comparing lamellar particles, such as the ones obtained in this work. ${ }^{48}$ Samples were also characterized in terms of Zeta potential ( $\zeta$ potential), with the same instrument.

Mathematical modelling. The adsorption results were fitted to six different adsorption isotherms models: Langmuir, Freundlich, Tempkin, Redlich-Peterson, Sips and Brunauer-Emmett-Teller. The constants of each model were determined by non-linear regression by using the Solver Add-in of Microsoft Excel®. The equations 
corresponding to each model, a detailed description thereof and the procedure applied for the estimation of their constants are presented in Supporting Information.

\section{Results and discussion}

\section{Synthesis of macroRAFT agents}

Three different types of macroRAFT agents, presenting anionic, cationic or non-ionic (linear or pending) blocks, were synthesized by solution polymerization which represents the initial step towards the preparation of polymer/Laponite nanocomposite latexes by the REEP process. As mentioned previously, these (co)polymers have either AA-, DMAEMA- or PEG-based structures, and the presence of each of these repeating units aims to tune the interaction between the macroRAFT agents and Laponite. Ionizable DMAEMA units, with opposite charge to that of the inorganic particle surface, were strategically chosen to promote strong electrostatic interaction of the macroRAFT copolymers with the negatively charged clay particles. ${ }^{49-52}$ As an alternative to cationic macroRAFT agents, PEG-based homo and copolymers were also selected considering their potential to adsorb on clay particles. ${ }^{47,53-57}$ When compared to positively charged molecules, a weaker interaction is expected for neutral PEG polymers, so some of these molecules were also designed containing negatively charged carboxylic acid groups (AA) to tune the interaction of the macroRAFT agents with the inorganic surface due to a potential interaction with the positively charged rims of the platelets as well. In addition, copolymers containing random units of BA were designed, aiming to increase the hydrophobicity of the clay environment, which is highly hydrophilic, in order to attract, in the case of a subsequent REEP, the growing hydrophobic polymeric block close to the Laponite/macroRAFT domains. Both AA- 
and DMAEMA-based polyelectrolytes as well as the pending or linear polar blocks of ethylene glycol, are also expected to provide stability to the hybrid particles. The main structures synthesized, as well as the RAFT agent, monomers and mPEG used in the synthesis, are represented in Figure 1.

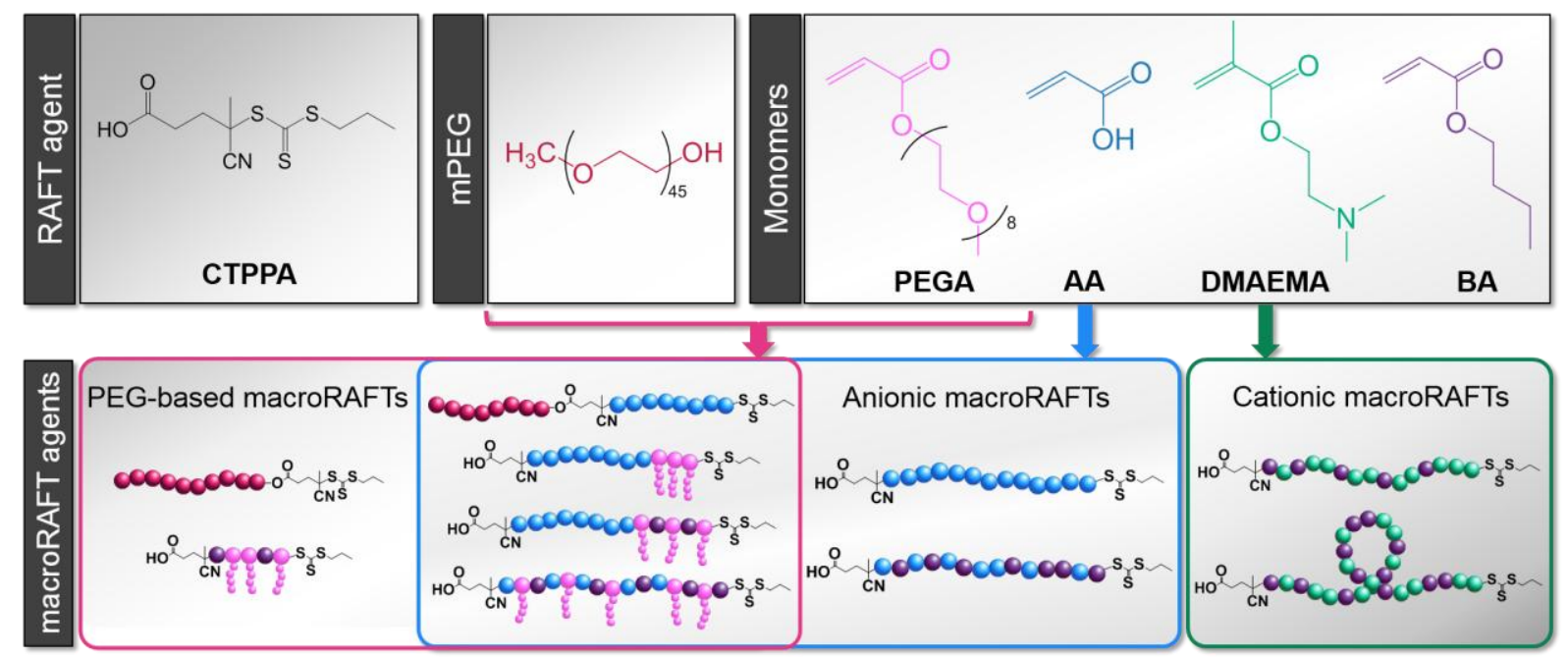

Figure 1. CTPPA RAFT agent, $\mathrm{mPEG}$ and monomers used in the synthesis of the macroRAFT agents employed in the adsorption study with Laponite and schematic representation of the different macroRAFT structures synthesized.

The results of overall monomer conversion $(X)$, theoretical and experimental molar mass $\left(M_{\mathrm{n}}\right.$, theo, $\left.M_{\mathrm{n}, \exp }\right)$ and dispersity $(\bigoplus)$ of all macroRAFT agents synthesized in this work are listed in Table 1. Polymerizations were successfully carried out and the synthesis of most macroRAFT agents followed a controlled behavior, according to the RAFT mechanism. The polymers synthesized, which were further used in the adsorption experiments, were well defined in terms of microstructure, composition, chain length and dispersity (more detailed information can be found in the Supporting Information). Since the use of PMMA standards for SEC analysis and the presence of residual macromonomer and methylation agent in some samples prejudiced the obtainment of reliable experimental $M_{\mathrm{n}}$ values for polymers with low molar 
mass, a deviation from theoretical $M_{\mathrm{n}}$ could be observed in some cases. However, the homo and copolymers synthesized were considered adequate for the study proposed in this work. ${ }^{14}$

\section{Adsorption isotherms of anionic macroRAFTs onto Laponite}

To better understand the interactions between AA-based macroRAFT agents and Laponite, the adsorption behavior of the $\mathrm{PAA}_{40}-\mathrm{CTPPA}(\mathrm{MR} 1)$ and $\mathrm{P}\left(\mathrm{AA}_{16}-\mathrm{co}-\mathrm{BA}_{16}\right)$ CTPPA (MR2) macroRAFT agents onto Laponite was studied. The adsorption of negatively charged (co)polymers on negative charged clay surface is usually very low. It has been reported that the adsorption of PAA (50000 $\left.\mathrm{g} \mathrm{mol}^{-1}\right)$ on sodium MMT at $\mathrm{pH} 5.6$ resulted in maximum adsorption capacity of $6.75 \mathrm{mg} \mathrm{g}^{-1}\left(0.135 \mu \mathrm{mol} \mathrm{g}{ }^{-1}\right),{ }^{58}$ while the adsorption of PAA $\left(3400 \mathrm{~g} \mathrm{~mol}^{-1}\right.$ ) on Kaolinite at $\mathrm{pH} 7$ resulted in a $q_{\max }$ value of $1.73 \mathrm{mg} \mathrm{g}^{-1}$ (i.e., $\left.0.51 \mu \mathrm{mol} \mathrm{g}^{-1}\right) .{ }^{59}$ In this work, the adsorption isotherms of the anionic macroRAFT agents, as well as all macroRAFT agents containing both AA and PEGA units, were carried out at a fixed $\mathrm{pH}$ of 7.5. This $\mathrm{pH}$ was selected since it is high enough to guarantee the ionization of the carboxyl groups of the AA units, and hence the solubility of the macroRAFT agents in water, including those containing BA units. In addition, at this $\mathrm{pH}$, the groups located at the edges of the platelets are protonated causing them to be positively charged. The results shown in Figure 2 reveal that the adsorption of MR1 on Laponite at $\mathrm{pH} 7.5$ is low. A reasonable explanation for the poor interaction between Laponite and PAA could rely on the fact that, under the experimental conditions used, the overall surface charge of the clay is negative, and it leads, therefore, to the formation of a repulsive force between the clay and the also negatively charged AA moieties. It is possible that the edges of the platelets exhibit, in fact, a negative electrostatic field that prevails over their positive charge due to the spilling over of the negative double layer from the faces into the edge region. This 
phenomenon, known as the spillover effect, has been described by Secor et al. ${ }^{60}$ as an important event affecting anion adsorption on MMT platelets at low electrolyte concentration, and it depends on the aspect ratio of the particles, the charge density of the edges and the ionic strength of the medium. In the case of MMT, it has been shown that the presence of negatively charged AA units in the macroRAFT agent structure, as compared to a similar AA-free structure, leads to a lower affinity of the macroRAFT for the clay surface. ${ }^{42}$ Indeed, the larger aspect ratio of the MMT particles and the lower charge density of their edges may make them more susceptible to the spillover effect than Laponite. Although less pronounced than for MMT, in the present case, the existence of repulsive electrostatic forces emanating from the particle can also possibly prevent anion adsorption onto the edges of the clay resulting in low MR1 adsorption.

On the other hand, a considerable higher adsorbed amount of $\mathrm{P}\left(\mathrm{AA}_{16}-\right.$ co- $\left.\mathrm{BA}_{16}\right)$ CTPPA (MR2), compared to PAA $40^{-C T P P A ~(M R 1), ~ c a n ~ b e ~ o b s e r v e d, ~ i n d i c a t i n g ~ t h e ~}$ importance of the presence of hydrophobic BA units for adsorption. ${ }^{61,62}$ Although the mechanism that drives the interaction between amphipathic copolymers and hydrophilic surfaces is not very obvious, ${ }^{63,64}$ the poor solubility of BA in water can be considered as one of the explanations to the increase in adsorption. Hydrophobic units can adsorb onto Laponite surface in order to minimize their interaction with the aqueous medium. ${ }^{42,61}$ The random distribution of the BA units along the copolymer chains guarantees that the later do not self assemble into micelles, and are therefore not shielded from water by the hydrophilic groups, promoting adsorption. Since the

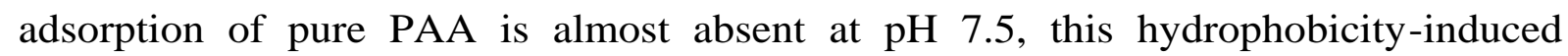
interaction might represent the main driving force for adsorption in the case of the MR2 copolymer. Similar hydrophobic effects have already been reported in the literature for the adsorption of analogous macroRAFT copolymers onto LDH 
platelets. ${ }^{65}$ Silva et al. $^{42}$ also showed that the presence of BA units along the macroRAFT agent structure influenced adsorption. However, in this previous work, the presence of PEGA units in the same structure did not allow this effect to be isolated, and thus conclusively demonstrated.

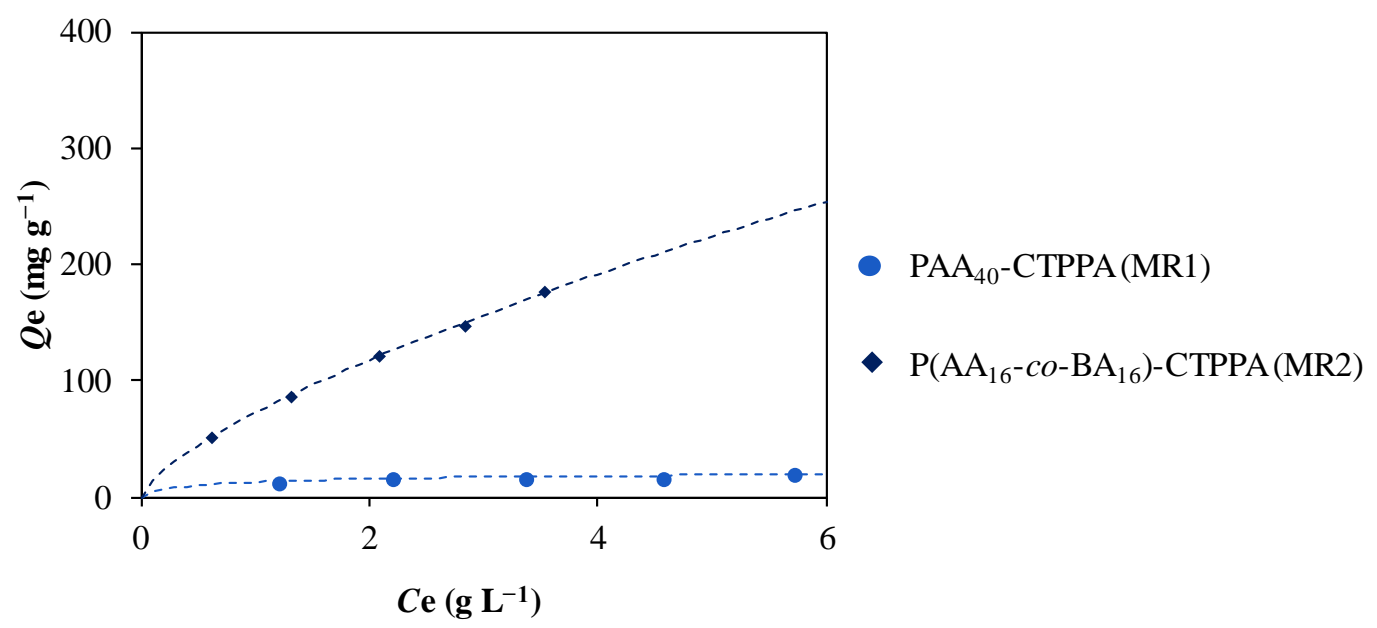

Figure 2. Equilibrium adsorption isotherms of AA-based macroRAFT agents onto the Laponite surface at $\mathrm{pH}=7.5$. Laponite concentration $=5.0 \mathrm{~g} \mathrm{~L}^{-1} \cdot \bullet=\mathrm{PAA}_{40} \mathrm{CTPPA}(\mathrm{MR} 1)$ and $\bullet=\mathrm{P} \mathrm{AA}_{16^{-}}$ co-BA ${ }_{16}$ )-CTPPA (MR2). The dashed lines represent the best fitting to the Redlich-Peterson adsorption model.

The adsorption isotherms were fitted to the Langmuir, Freundlich, Tempkin, RedlichPeterson, Sips and BET models (see Supporting Information for details). According to Tables S2 and S3 (ESI†), the Redlich-Peterson model provided the highest coefficient of determination $\left(R^{2}\right)$. This isotherm combines both Langmuir and Freundlich isotherms into an empirical equation, approaching the Freundlich equation at high adsorbate concentration and being reduced to the Langmuir isotherm for $\beta=1$ $(0<\beta \leq 1)$, and was selected to fit the data in Figure 2. The Redlich-Peterson constants are listed in Table S3. MR1 presents a $q_{\text {max,RP }}$ of $14 \mathrm{mg} \mathrm{g}^{-1}$, while for MR2 this value is $73 \mathrm{mg} \mathrm{g}^{-1}$, which corroborates the positive effect of the hydrophobic BA units on adsorption. 


\section{Adsorption isotherms of PEG-based macroRAFTs onto Laponite}

The adsorption behavior of five different macroRAFT agents composed of linear or pending PEG blocks was investigated. The potential of PEG-based homo and copolymers to adsorb on clays is well known in the literature, ${ }^{42,47,66-69}$ however, when compared to positively charged molecules, a weaker interaction is expected for neutral PEG polymers. For this reason, some of these molecules were also designed containing AA and/or BA units to tune their interaction with the inorganic surface and, in these cases, the adsorption study was carried out at $\mathrm{pH} 7.5$ (i.e., below the edge isoelectric point, IEP), while the other isotherms were carried out at $\mathrm{pH} 10$. The adsorption isotherms are displayed in Figure 3.

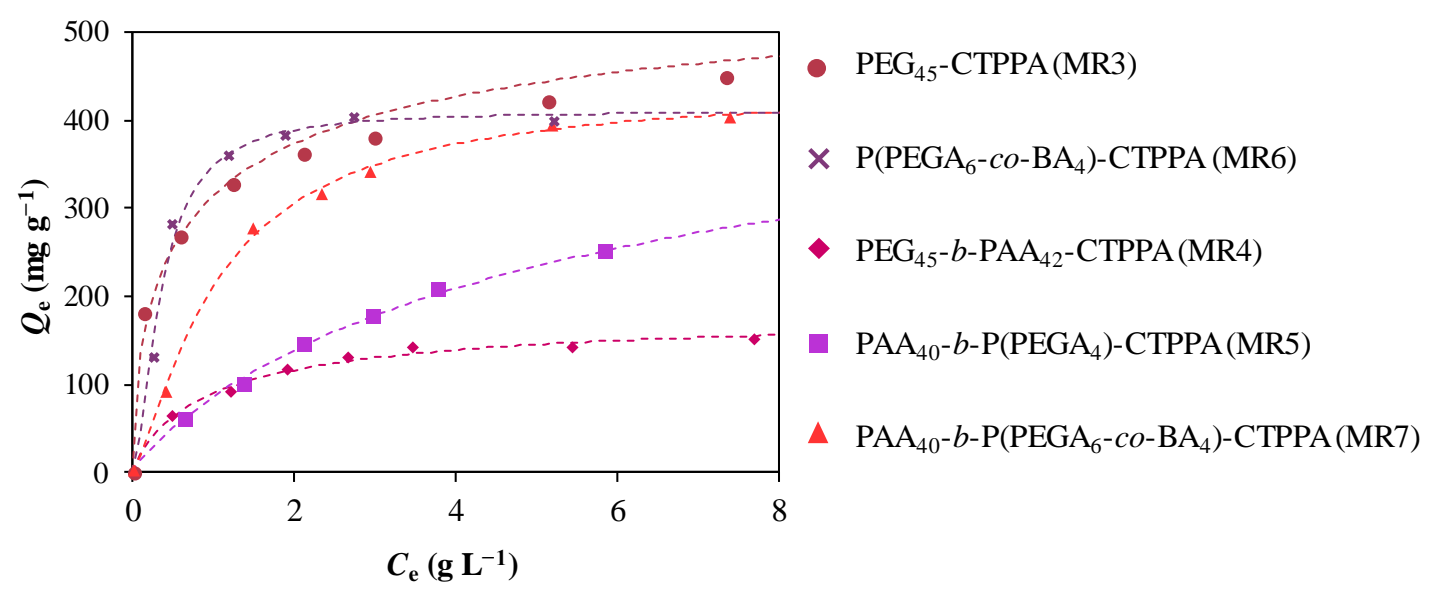

Figure 3. Equilibrium adsorption isotherms of PEG-based macroRAFT agents onto the Laponite surface. Laponite concentration $=5.0 \mathrm{~g} \mathrm{~L}^{-1} \cdot \bullet=\mathrm{PEG}_{45}-\mathrm{CTPPA}(\mathrm{MR} 3)$ at $\mathrm{pH} 10 ;$ CTPPA (MR4) at $\mathrm{pH} 7.5 ; \quad=\mathrm{PAA}_{40}-b-\mathrm{P}\left(\mathrm{PEGA}_{4}\right)-\mathrm{CTPPA}(\mathrm{MR} 5)$ at $\mathrm{pH} 7.5 ; \times=\mathrm{P}\left(\mathrm{PEGA}_{6}-c o-\mathrm{BA}_{4}\right)-$ CTPPA (MR6) at $\mathrm{pH} 10$ and $\Delta=\mathrm{PAA}_{40}-b-\mathrm{P}\left(\mathrm{PEGA}_{6}-c o-\mathrm{BA}_{4}\right)$-CTPPA (MR7) at $\mathrm{pH} 7.5$. The dashed lines represent the best fitting to the Sips adsorption model.

The isotherms of $\mathrm{PEG}_{45}-\mathrm{CTPPA}(\mathrm{MR} 3)$ and $\mathrm{P}\left(\mathrm{PEGA}_{6}-\mathrm{co}-\mathrm{BA}_{4}\right)-\mathrm{CTPPA}(\mathrm{MR} 6)$ display a steep initial slope that indicates that both non-ionic macroRAFTs present a strong interaction with the clay and a high affinity for the Laponite surface. In both cases, a 
clear plateau can be observed, which indicates that, with increasing the concentration of macroRAFT, the surface of Laponite becomes saturated and there is no further adsorption. Additionally, since the plateau is reached at high concentrations of adsorbed macroRAFTs, it can be concluded that the macroRAFTs allowed a high coverage of Laponite surface. According to the Sips model, the isotherm of MR3 displayed a maximum adsorption capacity of $604 \mathrm{mg} \mathrm{g}^{-1}\left(168 \mu \mathrm{mol} \mathrm{g} \mathrm{g}^{-1}\right)$ in agreement with previous literature, ${ }^{47}$ while for MR6 the value was $411 \mathrm{mg} \mathrm{g}^{-1}\left(142 \mu \mathrm{mol} \mathrm{g}{ }^{-1}\right)$. It is worth noting that similar $q_{\max }$ values have already been reported in the literature for the adsorption of $\mathrm{P}(\mathrm{PEGA}-\mathrm{co}-\mathrm{BA})-\mathrm{CTPPA}$ onto MMT at $\mathrm{pH} 8,{ }^{42}$ indicating that the interaction between the $\mathrm{P}(\mathrm{PEGA}-\mathrm{co}-\mathrm{BA})$ macroRAFT chains and the clay is not significantly influenced by the particles' different CEC values and aspect ratios.

The difference of adsorbed amounts between the two polymers, as to it, can be reasonably attributed to different conformations of adsorption. Indeed, linear non-ionic polymers (like MR3) adsorb on hydrophilic surfaces in the form of trains, loops and tails. The amount of loops is expected to depend on the molar mass of the polymers, and the formation of loops enables a closer packing of the chains at the surface, leading to an increase in the adsorbed amount. ${ }^{67}$ Comb-like polymers, on the other hand, have the ability to attach to surfaces not only through the side chains but also through the backbone segments. For this reason, these polymers adsorb onto surfaces in an extended conformation, lying flat on the inorganic surface and occupying a larger area. They are expected to present, therefore, lower adsorption in mass than the linear equivalents of comparable molar mass. ${ }^{70,71,72,73}$ The relatively strong adsorption observed in the case of MR6, can be thus considered to be the result of the interplay between: i) the effect of the pending chains (which depends on the conformation of the brush-like polymer in solution, and consequently on the length of the side chains with 
respect to the length of the backbone and on the number of side chains), ii) the effect of the slightly higher molar mass presented by this copolymer, and iii) the effect of the presence of hydrophobic BA units.

As shown in Table S2 and S3 (ESI†), all isotherms could be well fitted to the Sips model and the $q_{\max }$ values calculated by this model are all in good agreement with the maximum adsorption capacities determined experimentally.

Concerning the AA-containing PEG-based macroRAFTs, all of macroRAFTs belonging to this group present a lower affinity for Laponite than the corresponding AA-free polymers. When comparing the adsorption isotherm of $\mathrm{P}\left(\mathrm{PEGA}_{6}-\right.$ co- $\left.\mathrm{BA}_{4}\right)$ CTPPA (MR6) with the one of $\mathrm{PAA}_{40}-b-\mathrm{P}\left(\mathrm{PEGA}_{6}-c o-\mathrm{BA}_{4}\right)-\mathrm{CTPPA}$ (MR7), for example, different adsorption behaviors can be noticed with considerably lower affinity of the AA-containing molecule (MR7) for the Laponite surface, as indicated by its more gradual initial slope, at low $C_{\mathrm{e}}$ values. This lower affinity caused by the presence of the PAA block could be expected since the PAA block of MR7 should not interact with the clay, as previously discussed for the PAA-CTPPA macroRAFT agent (Figure 2), remaining instead dangling in solution while the P(PEGA-co-BA) block adsorbs on the clay surface. Indeed, the comparison between the adsorption behavior of MR7 and MR6 shows that, when expressed in $\mu \mathrm{mol} \mathrm{g}^{-1}$, the $q_{\mathrm{max}, \mathrm{S}}$ value is lower for MR7 (71 $\left.\mathrm{mol} \mathrm{g}^{-1}\right)$ than for MR6 (142 $\left.\mu \mathrm{mol} \mathrm{g}{ }^{-1}\right)$ but no effective difference can be noticed when these values are compared in $\mathrm{mg} \mathrm{g}^{-1}\left(438 \mathrm{mg} \mathrm{g}^{-1}\right.$ versus $411 \mathrm{mg} \mathrm{g}^{-1}$, respectively), which corroborates the aforementioned statements.

When comparing the adsorption isotherms of linear PEG $_{45}-$ CTPPA (MR3) and PEG $45^{-}$ $b$-PAA 42 -CTPPA (MR4), one can come to the same conclusion that AA hinders the adsorption of PEG units. Indeed, a considerable lower adsorption plateau can be

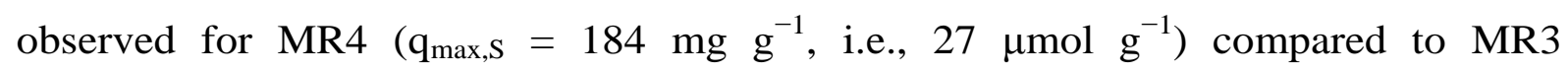


$\left(\mathrm{q}_{\max , \mathrm{S}}=604 \mathrm{mg} \mathrm{g}^{-1}\right.$, i.e., $\left.168 \mu \mathrm{mol} \mathrm{g}^{-1}\right)$. Nonetheless, when the PEG units were disposed in a pending conformation, as in the macroRAFT agent $\mathrm{PAA}_{40}-b-\mathrm{P}\left(\mathrm{PEGA}_{4}\right)-$ CTPPA (MR5), a higher adsorption was observed $\left(q_{\max , \mathrm{S}}=514 \mathrm{mg} \mathrm{g}^{-1}, 111 \mu \mathrm{mol} \mathrm{g}{ }^{-1}\right)$, when compared to $\mathrm{PEG}_{45}-b-\mathrm{PAA}_{42}$-CTPPA (MR4). It can be noticed that the isotherm did not reach a clear plateau in this case, which suggests multilayer adsorption. Indeed, while the PEG block is expected to adsorb on the clay surface, the PAA block would remain dangling in solution, in a tail-like conformation, available to interact with the PEGA units of new incoming macroRAFT agents, as it has already been described for MMT, for similar macroRAFT systems. ${ }^{42}$ Incrementing the non-charged segment with random units of $\mathrm{BA}$ in the macroRAFT $\mathrm{PAA}_{40}-b-\mathrm{P}\left(\mathrm{PEGA}_{6}-c o-\mathrm{BA}_{4}\right)-\mathrm{CTPPA}(\mathrm{MR} 7)$ seems to further promote adsorption, as already discussed for MR2, and the isotherm of this copolymer has a more defined equilibrium plateau, with a higher slope, in comparison to the isotherm of MR5 (PAA $\left.40-b-\mathrm{P}\left(\mathrm{PEGA}_{4}\right)-\mathrm{CTPPA}\right)$.

The adsorption of two random copolymers composed of AA, PEGA and BA with different molar masses was lastly evaluated. The isotherms of $\mathrm{P}\left(\mathrm{AA}_{4}-\right.$ co-PEGA $4-c o-$ $\left.\mathrm{BA}_{4}\right)$-CTPPA (MR8) and P(AA9-co-PEGA9-co-BA9)-CTPPA (MR9) are shown in Figure 4, as well as the isotherm of $\mathrm{PAA}_{40}-b-\mathrm{P}\left(\mathrm{PEGA}_{6}-c o-\mathrm{BA}_{4}\right)-\mathrm{CTPPA}(\mathrm{MR} 7)$, which is recalled from Figure 3. The dashed lines represent the fitting of the isotherms to the Sips equation, and the Sips constants obtained by non-linear regression are listed in Table S3 (ESI $\dagger)$. 


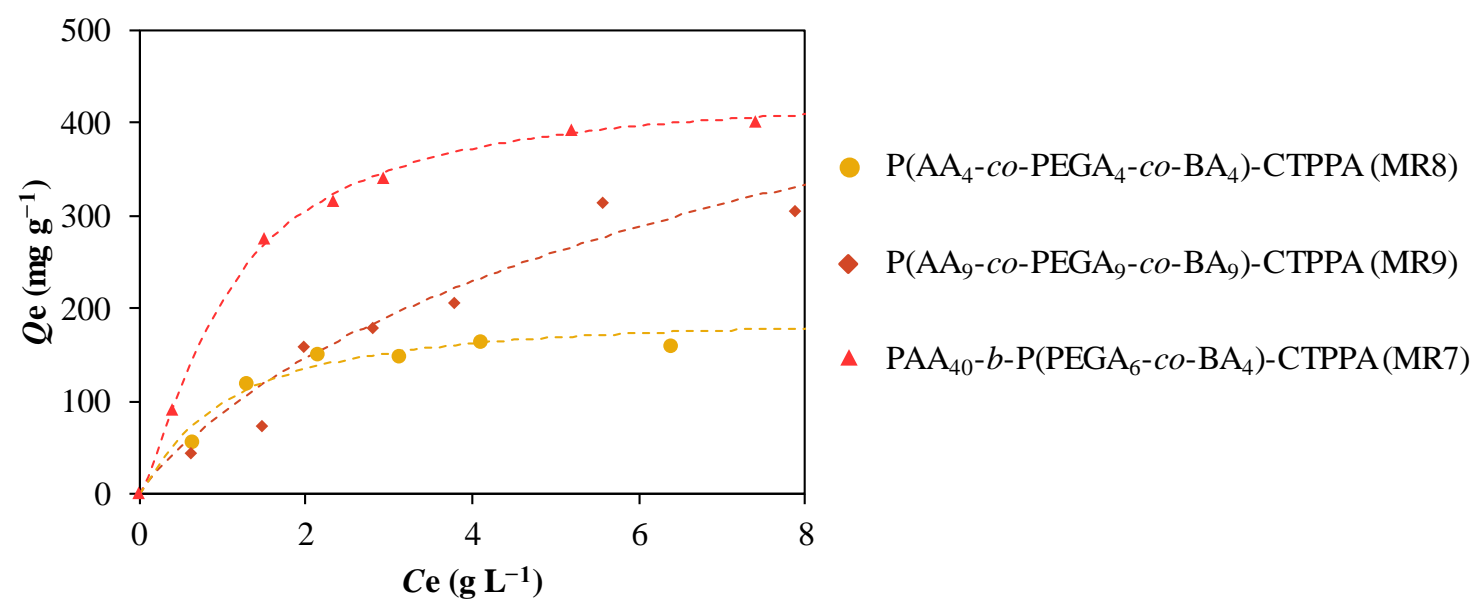

Figure 4. Equilibrium adsorption isotherms of: $\bullet=\mathrm{P}\left(\mathrm{AA}_{4}-c o-\mathrm{PEGA}_{4}-c o-\mathrm{BA}_{4}\right)-\mathrm{CTPPA}(\mathrm{MR} 8) ;$

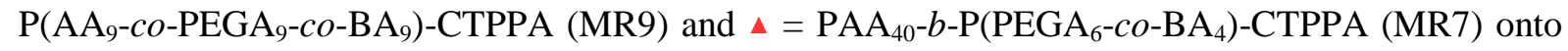
the Laponite surface at $\mathrm{pH}$ 7.5. Laponite concentration $=5.0 \mathrm{~g} \mathrm{~L}^{-1}$. The dashed lines represent the best fitting to the Sips adsorption model.

One might expect that the chain length has a great effect on polymer adsorption, since high molar mass polymers are more prone to give rise to longer tails and loops than smaller molecules, increasing the coverage of the particles and the affinity of the polymers for the clay surface (indicated by the initial slope). ${ }^{73}$ Indeed, in the case of MR8 and MR9, at high concentrations of macroRAFT, the adsorption of the longer

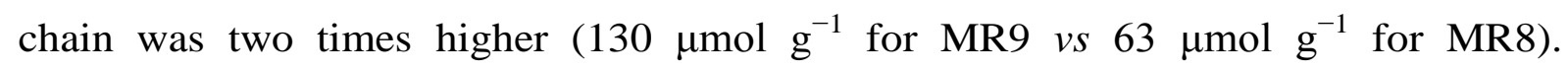
However, this polymer had only a moderate affinity for Laponite as indicated by the shape of the curve and the low initial slope, which can likely be ascribed to the higher number of AA units. Next, when comparing the adsorption isotherm of MR8 with that of the block copolymer MR7 $\left(q_{\text {max,S }}=71 \mu \mathrm{mol} \mathrm{g}{ }^{-1}\right)$, the higher affinity of the block copolymer is emphasized although MR7 contains more AA units, and indicates that the random distribution of $\mathrm{AA}$ along the copolymer chain is unfavorable for adsorption. A similar behaviour has been reported for the adsorption of random anionic copolymers on MMT clay at $\mathrm{pH} 8 .^{42}$ In that case, the adsorbed amount significantly increased at $\mathrm{pH} 5$ as the ionized AA units could then undergo electrostatic interactions with the 
MMT edges. The lower ionization degree of the macroRAFT agents at this $\mathrm{pH}$, may have also decreased electrostatic repulsions between the chains, promoting adsorption. However, in the case of Laponite, the use of a $\mathrm{pH}$ as low as 5 is not suitable due to gel formation. Yet, the analysis of the experimental adsorption data indicates that the values of maximum adsorption obtained for Laponite at $\mathrm{pH} 7.5$ are comparable to the ones obtained for MMT under similar $\mathrm{pH}$ conditions.

\section{Adsorption isotherms of cationic macroRAFTs onto Laponite}

Since Laponite is a cationic exchanger, polycations, such as the protonated form of PDMAEMA, can adsorb on its surface by electrostatic interaction between the opposite charges of the polyelectrolytes and the platelet surface. Thus, the incorporation of DMAEMA units in the macroRAFT agent is strategic for the promotion of strong electrostatic interactions between the macroRAFT and the negatively charged clay particles. Considering this, two different cationic macroRAFT agents based on DMAEMA (MR10 and MR11) were synthesized and submitted to a quaternization process to guarantee the total ionization of the chains independently of the $\mathrm{pH}$ of the medium. These macroRAFT agents were named MR10q and MR11q. They were used in the adsorption study as well as the unquaternized MR11. To ensure the ionization of MR11, its adsorption on Laponite was carried out at $\mathrm{pH} 6$ as the $\mathrm{p} K_{\mathrm{a}}$ of DMAEMA homo and copolymers is $\sim 7-8 . .^{74,} 75$ As the quaternized-based macroRAFT agents possess permanent positive charges, their adsorption was investigated at $\mathrm{pH}$ 10. At this $\mathrm{pH}$ value, the quaternized cationic macroRAFT agents could also adsorb on the possibly negatively charged edges of Laponite, ${ }^{76}$ which should thus guarantee the full coverage of the platelets. 
The adsorption results for all DMAEMA-based macroRAFT agents are shown in Figure 5. As expected for the adsorption of oppositely-charged polymers on clay minerals, high-affinity type isotherms (H-type, according to the Gilles classification ${ }^{77}$ ) were obtained for all DMAEMA-based macroRAFT agents. The isotherms were fitted to the adsorption models and a good fit was obtained for the three-parameter equations of Sips, Redlich-Peterson and BET models. However, some of the constants found for Sips and Redlich-Peterson, like $q_{\max }$, seems to be overestimated and therefore the BET model was chosen to represent the data. In addition, this model can describe both monolayer and multilayer adsorption. The fitting to the BET adsorption model is represented by the dashed lines in Figure 5, while the BET constants are listed in Table S3 (ESI $\dagger)$.

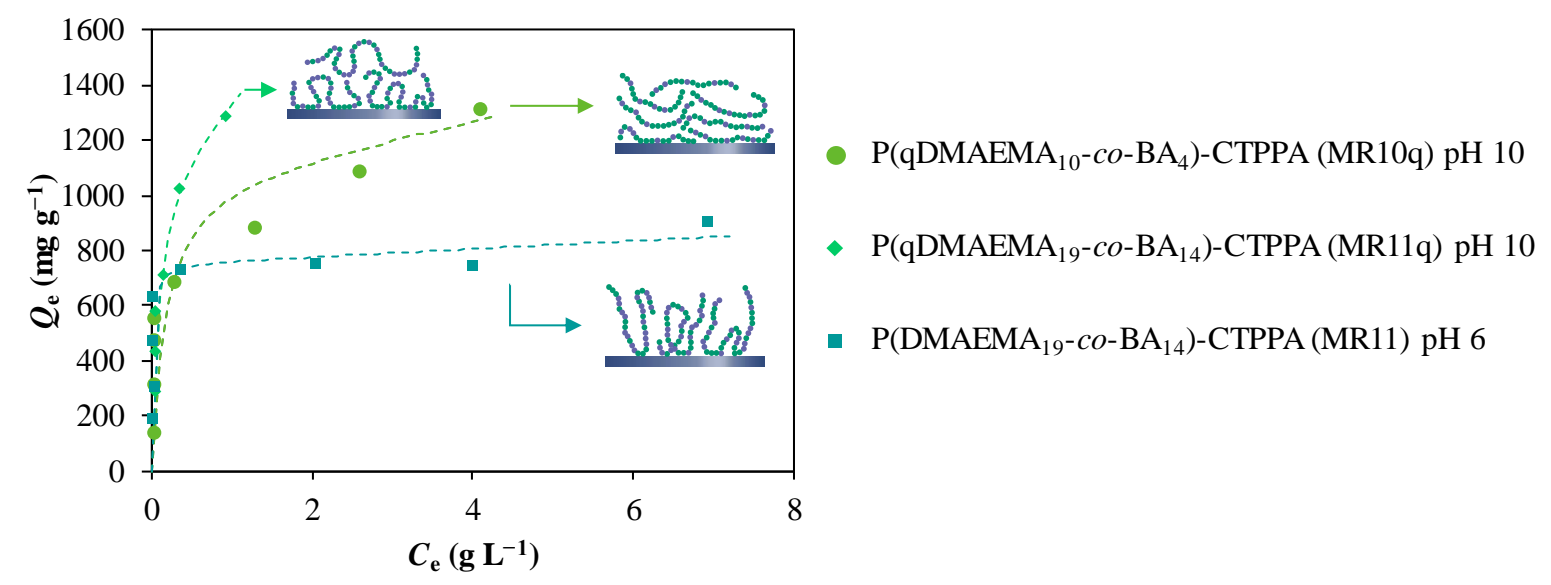

Figure 5. Equilibrium adsorption isotherms of cationic macroRAFT agents onto the Laponite surface. Laponite concentration $=5.0 \mathrm{~g} \mathrm{~L}^{-1} . \bullet=\mathrm{P}\left(\mathrm{qDMAEMA}_{10}-c o-\mathrm{BA}_{4}\right)-\mathrm{CTPPA}(\mathrm{MR} 10 \mathrm{q})$ at $\mathrm{pH} 10$;

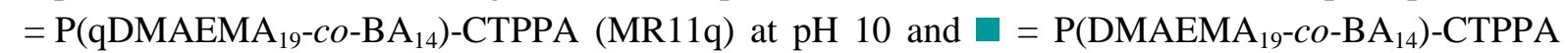
(MR11) at $\mathrm{pH}$ 6. The dashed lines represent the fitting to the BET adsorption model.

The shape of the isotherms, with steep slopes, indicates strong affinity of all macroRAFT agents for the clay surface, even for very low polymer concentrations. Indeed, due to electrostatic interactions, H-type isotherms are generally observed for 
the adsorption of polycations on clays, such as MMT. ${ }^{42}$ In Figure 5, it can be observed that the adsorption isotherms of the quaternized molecules, MR10q and MR11q, do not reach a clear plateau. Interestingly, the adsorption isotherm of MR11, the nonquaternized equivalent of MR11q, displays a well-defined plateau, indicating saturation of the surface, without further adsorption of polymer molecules onto the previously adsorbed polymer layer. The $q_{\max , \mathrm{B}}$ obtained using the BET model was 755 $\mathrm{mg} \mathrm{g}^{-1}$ (i.e., $167 \mu \mathrm{mol} \mathrm{g}^{-1}$ ), which agrees well with the maximum adsorption capacity determined experimentally $\left(163 \mu \mathrm{mol} \mathrm{g}^{-1}\right)$. In contrast, the strong interaction between the quaternized macroRAFT and Laponite results in a great amount of "trains" (adsorbed segments), leading to a flat conformation of the adsorbed chains, as mentioned earlier, in which the negative surface charges of the clay are neutralized by the DMAEMA units. In such circumstances, free ionized segments, such as loops, are scarce and the approaching of the macroRAFT chains in solution (and, therefore, the formation of bi- or multilayers of adsorbed polycations) is encouraged as illustrated in the inset of Figure 5. In addition, the intramolecular repulsive interaction between the charged units may force the chains to become more stretched and rigid, encouraging their adsorption in a flat conformation. This conformation, which predominates for moderately to highly charged polyelectrolytes, favors the formation of bi- or multilayers, resulting in higher adsorbed amounts at saturation. ${ }^{49}$ In this case, the presence of hydrophobic BA units along the chains may have additionally facilitated the formation of subsequent adsorbed layers by stimulating the contact between neighboring chains. The increase in the interchain contact can be noticed by the decrease in the values of $K_{\mathrm{B} 1}$ and increase of $K_{\mathrm{B} 2}$ of MR11q $\left(10.2\right.$ and $0.167 \mathrm{~L} \mathrm{mg}^{-1}$, respectively), as compared to MR11. In the case of non-quaternized MR11, the formation of multilayers was hindered by the adsorption of macroRAFT in a more 
extended conformation, which favors the formation of loops and tails that impose a barrier (by electrostatic repulsion) to the approaching of the macroRAFT that is in solution. For the adsorption of a similar MR structure on $\mathrm{MMT}^{42}{ }^{42}$ a higher adsorbed mass of polycations was equally observed for the quaternized structure in comparison to the non-quaternized molecules and attributed to the flatter conformation that the former acquired on the surface of the solids, which favored multilayer adsorption. However, the isotherms of the non-quaternized copolymers did not display welldefined plateaus, unlike what is observed here for Laponite. Therefore, some other differences between the two systems should be considered. First, the CEC of MMT (i.e., $\left.0.92 \mathrm{meq}^{-1}\right)$ is higher than that of Laponite $\left(0.75 \mathrm{meq} \mathrm{g}^{-1}\right)$, meaning that the platelets are more negatively charged and, at pH 5 (which is the case of MMT system) the macroRAFTs are more ionized and the affinity between them and MMT is stronger. Thus, chains may have laid flatter on the surface of MMT, encouraging further adsorption over the first layer. In addition, in the case of Laponite, as mentioned above, the $\mathrm{pH}$ of the untreated macroRAFT complex was adjusted to 6 and, due to the higher point of zero charge of Laponite edges and its smaller surface area, in comparison to MMT, an electrostatic repulsion between the edges of Laponite and the also positively charged molecules may have inhibited multilayer adsorption. At a higher $\mathrm{pH}$ of $\sim 10$, which was the case for the quaternized copolymer, the edges of the platelets are more likely to be negatively charged, and consequently not opposing adsorption, so a higher value of $q_{\mathrm{max}, \mathrm{B}}$ was obtained for the quaternized counterpart (1198 $\mathrm{mg} \mathrm{g}^{-1}$, i.e., $250 \mu \mathrm{mol} \mathrm{g}{ }^{-1}$ ). When both quaternized macroRAFT agents (MR10q and MR11q) are compared in terms of adsorption, a lower adsorbed amount at saturation is achieved for the shorter and less hydrophobic copolymer P(qDMAEMA $\left.10^{-} c o-\mathrm{BA}_{4}\right)-\mathrm{CTPPA}(\mathrm{MR} 10 \mathrm{q})$. Two main factors determine that the 
chain conformation is flatter in this system than it is for the adsorption of $\mathrm{P}\left(\mathrm{qDMAEMA}{ }_{19}-\mathrm{co}-\mathrm{BA}_{14}\right)$-CTPPA (MR11q). The first one is the lower molar mass of the chains. As mentioned earlier for the non-ionic macroRAFT agents, with decreasing the chain length, the formation of loops and tails is reduced, and the fraction of polymer units effectively adsorbed onto the mineral surface increases. The second factor is that MR10q has fewer hydrophobic units of BA, therefore, the cationicity and, consequently, the electrostatic repulsion between identically charged groups along the same chain is higher for MR10q than for MR11q. As the cationicity of the macroRAFT increases (either from higher degrees of ionization/quaternization or lower ionic strength), the formation of long loops and tails is inhibited, and a flat conformation of the polyelectrolyte is preferred. Moreover, as MR10q is poorer in BA units, it is less flexible than MR11q to adopt an extended conformation with loops. ${ }^{78}$ Even though the monolayer adsorption capacities of MR10q and MR11q are very similar $\left(q_{\max , \mathrm{B}}=1116\right.$ and $1198 \mathrm{mg} \mathrm{g}^{-1}$, respectively $)$, a comparison between the adsorption equilibrium constants of the first layer $\left(K_{\mathrm{B} 1}=5.7\right.$ for $\mathrm{MR} 10 \mathrm{q}$ and $10.2 \mathrm{mg}$ $\mathrm{g}^{-1}$ for MR11q) indicates that the adsorption of the first layer is favored in the case of MR11q. It is worth reminding that, despite the flatter conformation presented by MR10q, MR11q is richer in BA units and hydrophobic interactions play an important role in the adsorption of the first layer. A similar logic can be used when analyzing the adsorption equilibrium constants of the upper layers. The higher value obtained for $\operatorname{MR} 11 \mathrm{q}\left(K_{\mathrm{B} 2}=0.167 \mathrm{~L} \mathrm{mg}^{-1}\right)$ against MR10q $\left(K_{\mathrm{B} 2}=0.038 \mathrm{~L} \mathrm{mg}^{-1}\right)$ can be attributed to the more hydrophobic nature of this copolymer that causes intermolecular interactions and consequently facilitates the formation of multilayers. In both cases, the adsorption would be the result of the compromise between the formation of loops and tails and the hydrophobicity of the chains. 
For the successful synthesis of nanocomposite materials with controlled morphologies through the REEP technique, an efficient control over the initial dispersion of the macroRAFT/Laponite particles is a key factor, so it is crucial to guarantee that particles are well dispersed after the addition of the macroRAFT agent. Therefore, the evolution of zeta potential, the average hydrodynamic diameters and PdIs with the concentration of MR10q was evaluated, as shown in Figure 6. The results indicate that the particles are totally neutralized (point of zero charge, IEP) at very low concentrations of copolymer $\left(0.88 \mathrm{~g} \mathrm{~L}^{-1}\right)$, situation characterized by a severe coagulation. In fact, maximum aggregation commonly occurs for $\zeta$ potentials close to zero. Therefore, at the IEP, the measured particle size increases dramatically, as seen in the peak of Figure 6B. Charge reversal then occurs upon further addition of macroRAFT, restoring colloidal stability. Similar results were obtained for the non-quaternized macroRAFT $\mathrm{P}\left(\mathrm{DMAEMA}_{19}-\mathrm{Co}-\mathrm{BA}_{14}\right)$-CTPPA, MR11, and the corresponding figure is shown in the Supporting information (Figure S8).

(A)

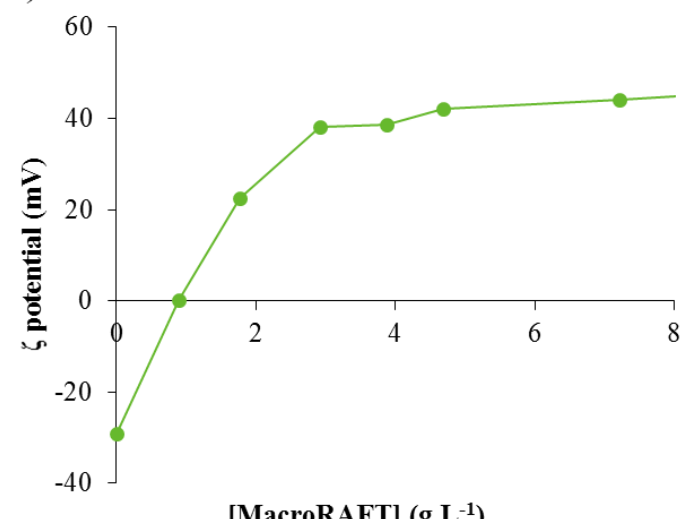

(B)

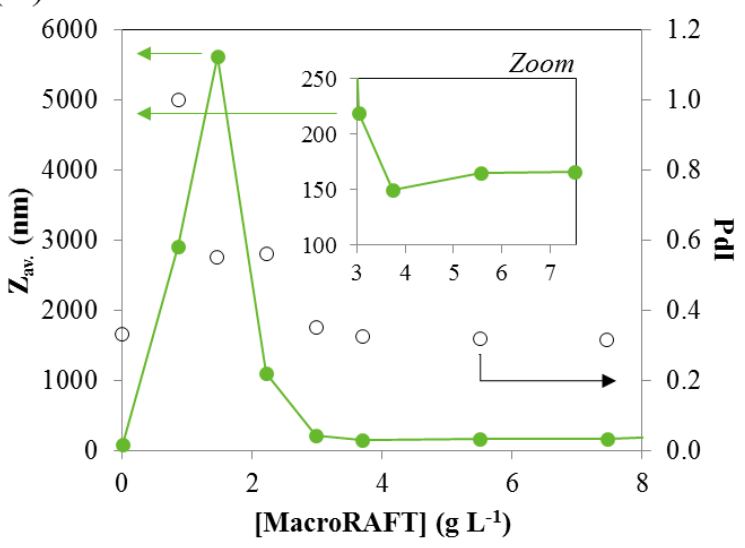

Figure 6. Evolution of (A) $\zeta$ potential and (B) average hydrodynamic diameters (- - ) and PdI (O) of Laponite platelets functionalized with different concentrations of MR10q at $\mathrm{pH} 10$. [Laponite] = $5 \mathrm{~g} \mathrm{~L}^{-1}$. 
At the point of zero charge, the macroRAFT agent presents a cationic charge of $0.99 \mathrm{meq} \mathrm{g}^{-1}$ of Laponite, which can be compared to the clay CEC $\left(0.75 \mathrm{meq} \mathrm{g}^{-1}\right.$, even though this value is an average value for the CEC of Laponite). The reversal of surface charges happens slightly above the CEC of Laponite, which can be explained by a discrete mismatch in charge separation between the cationic macroRAFT agent and the negative surface charges of Laponite (different average distances between negative charges in clay surface and between the quaternary nitrogen groups along the copolymer chains). ${ }^{73,79}$ This result could also be attributed to the adsorption of the macroRAFT chains preferentially in coil and loop-like rather than a complete flat conformation. Adsorption can continue even after the neutralization of the negative surface charges of Laponite, and the adsorbed amount at saturation $\left(q_{\max }\right)$, is considerably higher than the adsorbed amount found at the point of charge inversion. In fact, adsorption beyond the point of zero charge can be driven by hydrophobic interactions between the copolymer chains and by entropic effects (e.g., release of solvent and counterions from the charged copolymers), and has already been reported in the literature for a similar system involving LDH platelets and anionic macroRAFT agents. ${ }^{65,80}$ At elevated macroRAFT concentrations, the repulsive double-layer forces between the platelets (now positively charged, with platelets fully coated with macroRAFT chains) are re-established, leading to highly stable dispersions which is also consistent with previous literature. ${ }^{80}$

\section{Conclusions}

Equilibrium adsorption of AA-, PEGA- and DMAEMA-based RAFT homo and copolymers onto Laponite ${ }^{\circledR}$ RD has been studied by plotting the macroRAFT's solid 
phase against liquid phase concentrations in adsorption isotherms that were fitted to six different theoretical adsorption models. For PAA, it has been shown that there is possibly no effective interaction between these moieties and the positively charged edges of the clay, while its association with a linear block of PEG in the macroRAFT structure interferes in the adsorption process, although this effect seems to be reduced to some extent when ethylene glycol units are disposed as pending segments. The random disposition of AA units along the macroRAFT chain, rather than a block distribution, was also unfavorable for adsorption. Hydrophobic BA moieties, on the other hand, have been shown play an important role on adsorption, favoring macroRAFT-clay and macroRAFT-macroRAFT hydrophobic interactions and encouraging multilayer adsorption. The adsorption of cationic macroRAFT agents onto Laponite resulted in high-affinity-type curves. Indeed, a general comparison between cationic, non-ionic and anionic macroRAFT agents reveals a considerably higher affinity of the positively charged molecules for the surface of Laponite. Polyelectrolytes with higher cationicity presented higher tendency to adsorb in a flat conformation, which seems to facilitate multilayer adsorption, while higher cationicity facilitates the formation of loops and tails, which impede the approximation of other chains and result predominantly in monolayer adsorption. Indeed, the conformation that the adsorbed polymeric chains adopt on the inorganic surface seems to be crucial in the adsorption process, being determined by several factors, including the $\mathrm{pH}$ of the medium, the flexibility of the molecules, their cationicity, and their molar mass. In addition to these factors, the adsorption process is strongly influenced by some specific characteristics of the clay particles, such as the CEC and the edge IEP, as observed when Laponite and $\mathrm{MMT}^{42}$ isotherms were compared. The strong adsorption of cationic copolymers onto clay minerals makes them extremely attractive and promising 
for the synthesis of nanocomposites. Despite the challenge these molecules present in terms of colloidal stability due to coagulation and bridging effects of the polycations, the results obtained in this work show that stable systems can be obtained with both quaternized and non-quaternized DMAEMA-based macroRAFT agents. Uncharged macroRAFT agents PEG-CTPPA and P(PEGA-co-BA)-CTPPA are very interesting for nanocomposite synthesis as well, since they yield relatively strong adsorption resulting in high adsorbed amounts of macroRAFT agent. The effect of macroRAFT composition on the morphology of polymer/Laponite particles synthesized by REEP will be addressed in future publications.

\section{ASSOCIATED CONTENT}

Supporting Information. The following files are available free of charge. Adsorption isotherm models used to fit the experimental data, results for the synthesis of the macroRAFT agents; estimation of adsorption model parameters for adsorption of non-ionic, anionic and cationic macroRAFT agents onto Laponite; Evolution of $\zeta$ potential, average hydrodynamic diameters and PdI of Laponite platelets functionalized with MR10q and MR11; X-Ray diffraction (XRD) analysis of P(DMAEMA $\left.19-c o-\mathrm{BA}_{14}\right)-\mathrm{CTPPA} /$ Laponite dispersions. (PDF)

\section{Corresponding Author}

*E-mail: bourgeat@1ccp.cpe.fr (E.B-L.).

*E-mail: amsantos@usp.br (A.M.S.).

\section{Author Contributions}


The manuscript was written through contributions of all authors. All authors have given approval to the final version of the manuscript.

\section{Notes}

The authors declare no competing financial interest.

\section{Acknowledgements}

The authors gratefully acknowledge financial support from the funding agencies FAPESP (2010/19919-6), CNRS and FCT/MEC from Brazil, France and Portugal, respectively, and IUPAC for enabling the ENCIRCLE (Polymer Encapsulation of anisotropic inorganic particles by RAFT-mediated emulsion polymerization) project, of which this work was part. The authors thank Juliana Livi Antoniassi from LCT (Poli-USP) for XRD analysis.

\section{References}

1. Bourgeat-Lami, E.; Lansalot, M., Organic/inorganic composite latexes: the marriage of emulsion polymerization and inorganic chemistry. Adv. Polym. Sci. 2010, 233, 53-123.

2. Sanchez, C.; Julian, B.; Belleville, P.; Popall, M., Applications of hybrid organicinorganic nanocomposites. J. Mater. Chem. 2005, 15, 3559-3592.

3. Gomez-Romero, P., Hybrid organic-inorganic materials - In search of synergic activity. Adv. Mater. 2001, 13, 163-174.

4. Zetterlund, P. B.; Thickett, S. C.; Perrier, S.; Bourgeat-Lami, E.; Lansalot, M., Controlled/Living Radical Polymerization in Dispersed Systems: An Update. Chem. Rev. 2015, 115, 9745-9800.

5. Cenacchi-Pereira, A.; Grant, E.; D’Agosto, F.; Lansalot, M.; Bourgeat-Lami, E., Encapsulation with the Use of Controlled Radical Polymerization. In Encyclopedia of 
Polymeric Nanomaterials, Kobayashi, S.; Müllen, K., Eds. Springer Berlin Heidelberg: 2015; pp 718-729.

6. Bourgeat-Lami, E.; D’Agosto, F.; Lansalot, M., Synthesis of Nanocapsules and Polymer/Inorganic Nanoparticles Through Controlled Radical Polymerization At and Near Interfaces in Heterogeneous Media. Adv. Polym. Sci. 2016, 270, 123-161.

7. Qiao, X. G.; Lansalot, M.; Bourgeat-Lami, E.; Charleux, B., Nitroxide-Mediated Polymerization-Induced Self-Assembly of Poly(poly(ethylene oxide) methyl ether methacrylate-co-styrene)-b-poly(n-butyl methacrylate-co-styrene) Amphiphilic Block Copolymers. Macromolecules 2013, 46, 4285-4295.

8. Qiao, X. G.; Dugas, P. Y.; Charleux, B.; Lansalot, M.; Bourgeat-Lami, E., Synthesis of multipod-like silica/polymer latex particles via nitroxide-mediated polymerization-induced self-assembly of amphiphilic block copolymers. Macromolecules 2015, 48, 545-556.

9. Qiao, X. G.; Lambert, O.; Taveau, J. C.; Dugas, P. Y.; Charleux, B.; Lansalot, M.; Bourgeat-Lami, E., Nitroxide-Mediated Polymerization-Induced Self-Assembly of Block Copolymers at the Surface of Silica Particles: Toward New Hybrid Morphologies. Macromolecules 2017, 50, 3796-3806.

10. Qiao, X. G.; Dugas, P. Y.; Charleux, B.; Lansalot, M.; Bourgeat-Lami, E., Nitroxide-mediated polymerization-induced self-assembly of amphiphilic block copolymers with a pH/temperature dual sensitive stabilizer block. Polym. Chem. 2017, 8, 4014-4029.

11. Loiko, O. P.; Spoelstra, A. B.; van Herk, A. M.; Meuldijk, J.; Heuts, J. P. A., An ATRP-based approach towards water-borne anisotropic polymer-Gibbsite nanocomposites. Polym. Chem. 2016, 7, 3383-3391.

12. Nguyen, D.; Zondanos, H. S.; Farrugia, J. M.; Serelis, A. K.; Such, C. H.; Hawkett, B. S., Pigment encapsulation by emulsion polymerization using macro-RAFT copolymers. Langmuir 2008, 24, 2140-2150. 
13. Daigle, J.-C.; Claverie, J. P., A simple method for forming hybrid core-shell nanoparticles suspended in water. J. Nanomater. 2008, 2008, 1-8.

14. Das, P.; Zhong, W.; Claverie, J. P., Copolymer nanosphere encapsulated CdS quantum dots prepared by RAFT copolymerization: synthesis, characterization and mechanism of formation. Colloid Polym. Sci. 2011, 289, 1519-1533.

15. Das, P.; Claverie, J. P., Synthesis of single- core and multiple- core core- shell nanoparticles by RAFT emulsion polymerization: Lead sulfide- copolymer nanocomposites. J. Polym. Sci. Part A: Polym. Chem. 2012, 50, 2802-2808.

16. Zgheib, N.; Putaux, J.-L.; Thill, A.; Bourgeat-Lami, E.; D'Agosto, F.; Lansalot, M., Cerium oxide encapsulation by emulsion polymerization using hydrophilic macroRAFT agents. Polym. Chem. 2013, 4, 607-614.

17. Bourgeat-Lami, E.; França, A. J. P. G.; Chaparro, T. C.; Silva, R. D.; Dugas, P. Y.; Alves, G. M.; Santos, A. M., Synthesis of Polymer/Silica Hybrid Latexes by Surfactant-Free RAFT-Mediated Emulsion Polymerization. Macromolecules 2016, 49, 4431-4440.

18. Li, K.; Dugas, P.-Y.; Bourgeat-Lami, E.; Lansalot, M., Polymer-encapsulated $\gamma$ Fe2O3 nanoparticles prepared via RAFT-mediated emulsion polymerization. Polymer 2016, $106,249-260$.

19. Nguyen, D.; Pham, B. T.; Huynh, V.; Kim, B. J.; Pham, N. T.; Bickley, S. A.; Jones, S. K.; Serelis, A.; Davey, T.; Such, C., Monodispersed polymer encapsulated superparamagnetic iron oxide nanoparticles for cell labeling. Polymer 2016, 106, 238-248.

20. Zhong, W.; Zeuna, J. N.; Claverie, J. P., A versatile encapsulation method of noncovalently modified carbon nanotubes by RAFT polymerization. J. Polym. Sci. Part A: Polym. Chem. 2012, 50, 4403-4407.

21. Nguyen, D.; Such, C. H.; Hawkett, B. S., Polymer coating of carboxylic acid functionalized multiwalled carbon nanotubes via reversible addition-fragmentation chain 
transfer mediated emulsion polymerization. J. Polym. Sci. Part A: Polym. Chem. 2013, 51, 250-257.

22. Ali, S. I.; Heuts, J. P. A.; Hawkett, B. S.; van Herk, A. M., Polymer encapsulated gibbsite nanoparticles: efficient preparation of anisotropic composite latex particles by RAFTbased starved feed emulsion polymerization. Langmuir 2009, 25, 10523-10533.

23. Cenacchi, A.; Pearson, S.; Kostadinova, D.; Leroux, F.; D'Agosto, F.; Lansalot, M.; Bourgeat-Lami, E.; Prevot, V., Nanocomposite latexes containing layered double hydroxides via RAFT-assisted encapsulating emulsion polymerization. Polym. Chem. 2017, 8, 12331243.

24. Pearson, S.; Pavlovic, M.; Augé, T.; Torregrossa, V.; Szilagyi, I.; D’Agosto, F.; Lansalot, M.; Bourgeat-Lami, E.; Prévot, V., Controlling the Morphology of Film-Forming, Nanocomposite Latexes Containing Layered Double Hydroxide by RAFT-Mediated Emulsion Polymerization. Macromolecules 2018, 51, 3953-3966.

25. Dalmas, F.; Pearson, S.; Gary, B.; Chenal, J.-M.; Bourgeat-Lami, E.; Prevot, V.; Chazeau, L., Tailored microstructure and mechanical properties of nanocomposite films made from polyacrylic/LDH hybrid latexes synthesized by RAFT-mediated emulsion polymerization. Polym. Chem. 2018, 9, 2590-2600.

26. Mballa Mballa, M. A.; Ali, S. I.; Heuts, J. P. A.; van Herk, A. M., Control of the anisotropic morphology of latex nanocomposites containing single montmorillonite clay particles prepared by conventional and reversible addition- fragmentation chain transfer based emulsion polymerization. Polym. Int. 2012, 61, 861-865.

27. Silva, R. D.; Chaparro, T. d. C.; Monteiro, I. S.; Dugas, P.-Y.; D’Agosto, F.; Lansalot, M.; Martins dos Santos, A.; Bourgeat-Lami, E., Tailoring the Morphology of Polymer/Montmorillonite Hybrid Latexes by Surfactant-Free Emulsion Polymerization Mediated by Amphipathic MacroRAFT Agents. Macromolecules 2019, 52, 4979-4988. 
28. Huynh, V. T.; Nguyen, D.; Such, C. H.; Hawkett, B. S., Polymer Coating of Graphene Oxide via Reversible Addition-Fragmentation Chain Transfer Mediated Emulsion Polymerization. J. Polym. Sci. Part A: Polym. Chem. 2015, 53, 1413-1421.

29. Sinha Ray, S.; Okamoto, M., Polymer/layered silicate nanocomposites: a review from preparation to processing. Prog. Polym. Sci. 2003, 28, 1539-1641.

30. Okamoto, M., Recent advances in polymer/layered silicate nanocomposites: an overview from science to technology Mater. Sci. Technol. 2006, 22, 756-779.

31. Pavlidou, S.; Papaspyrides, C. D., A review on polymer-layered silicate nanocomposites. Prog. Polym. Sci. 2008, 33, 1119-1198.

32. Ray, S. S., Recent Trends and Future Outlooks in the Field of Clay-Containing Polymer Nanocomposites. Macromol. Chem. Phys. 2014, 215, 1162-1179.

33. Kotal, M.; Bhowmick, A. K., Polymer nanocomposites from modified clays: Recent advances and challenges. Prog. Polym. Sci. 2015, 51, 127-187.

34. Negrete-Herrera, N.; Putaux, J.-L.; Bourgeat-Lami, E., Synthesis of polymer/Laponite nanocomposite latex particles via emulsion polymerization using silylated and cationexchanged Laponite clay platelets. Progress in Solid State Chemistry 2006, 34, 121-137.

35. Mourchid, A.; Lecolier, E.; Van Damme, H.; Levitz, P., On viscoelastic, birefringent, and swelling properties of Laponite clay suspensions: Revisited phase diagram. Langmuir 1998, $14,4718-4723$.

36. Jatav, S.; Joshi, Y. M., Chemical stability of Laponite in aqueous media. Applied Clay Science 2014, 97-98, 72-77.

37. Cummins, H. Z., Liquid, glass, gel: the phases of colloidal Laponite. J. Non-Cryst. Solids 2007, 353, 3891-3905. 
38. Tombacz, E.; Szekeres, M., Colloidal behavior of aqueous montmorillonite suspensions: the specific role of $\mathrm{pH}$ in the presence of indifferent electrolytes. Applied Clay Science 2004, 27, 75-94.

39. Cadene, A.; Durand-Vidal, S.; Turq, P.; Brendle, J., Study of individual Namontmorillonite particles size, morphology, and apparent charge. J. Colloid Interface Sci. 2005, 285, 719-730.

40. Dijkstra, M.; Hansen, J. P.; Madden, P. A., Gelation of a clay colloid suspension. Phys. Rev. Lett. 1995, 75, 2236-2239.

41. Liu, Y.; Zhu, M. F.; Liu, X. L.; Zhang, W.; Sun, B.; Chen, Y. M.; Adler, H. J. P., High clay content nanocomposite hydrogels with surprising mechanical strength and interesting deswelling kinetics. Polymer 2006, 47, 1-5.

42. Silva, R. D.; Stefanichen Monteiro, I.; Chaparro, T. d. C.; Silva Hardt, R.; Giudici, R.; Barros-Timmons, A.; Bourgeat-Lami, E.; Martins dos Santos, A., Investigation of the Adsorption of Amphipathic macroRAFT Agents onto Montmorillonite Clay. Langmuir 2017, $33,9598-9608$.

43. Buetuen, V.; Armes, S. P.; Billingham, N. C., Selective quaternization of 2(dimethylamino)ethyl methacrylate residues in tertiary amine methacrylate diblock copolymers. Macromolecules 2001, 34, 1148-1159.

44. Zhao, W.; Fonsny, P.; FitzGerald, P.; Warr, G. G.; Perrier, S., Unexpected behavior of polydimethylsiloxane/poly(2-(dimethylamino)ethyl acrylate) (charged) amphiphilic block copolymers in aqueous solution. Polym. Chem. 2013, 4, 2140-2150.

45. Hashimoto, N.; Aoyama, T.; Shioiri, T., New methods and reagents in organic synthesis. 14. A simple efficient preparation of methyl esters with trimethylsilyldiazomethane (TMSCHN2) and its application to gas chromatographic analysis of fatty acids. Chem. Pharm. Bull. 1981, 29, 1475-1478. 
46. Stern, O., The theory of the electrolytic double-layer. Z. Elektrochem 1924, 30, 10141020.

47. Guimaraes, T. R.; Chaparro, T. d. C.; D'Agosto, F.; Lansalot, M.; dos Santos, A. M.; Bourgeat-Lami, E., Synthesis of multi-hollow clay-armored latexes by surfactant-free emulsion polymerization of styrene mediated by poly(ethylene oxide)-based macroRAFT/Laponite complexes. Polym. Chem. 2014, 5, 6611-6622.

48. Ploehn, H. J.; Liu, C., Quantitative Analysis of Montmorillonite Platelet Size by Atomic Force Microscopy. Ind. Eng. Chem. Res. 2006, 45, 7025-7034.

49. Durandpiana, G.; Lafuma, F.; Audebert, R., Flocculation and adsorption properties of cationic polyelectrolytes toward Na-montmorillonite dilute suspensions. J. Colloid Interface Sci. 1987, 119, 474-480.

50. Denoyel, R.; Durand, G.; Lafuma, F.; Audebert, R., Adsorption of cationic polyelectrolytes onto montmorillonite and silica - microcalorimetric study of their conformation. J. Colloid Interface Sci. 1990, 139, 281-290.

51. Churchman, G. J., Formation of complexes between bentonite and different cationic polyelectrolytes and their use as sorbents for non-ionic and anionic pollutants. Appl. Clay Sci. 2002, 21, 177-189.

52. Huang, X.; Xu, S.; Zhong, M.; Wang, J.; Feng, S.; Shi, R., Modification of Nabentonite by polycations for fabrication of amphoteric semi-IPN nanocomposite hydrogels. Appl. Clay Sci. 2009, 42, 455-459.

53. Parfitt, R. L.; Greenland, D. J., The adsorption of poly(ethylene glycols) on clay minerals. Clay Miner. 1970, 8, 305-315.

54. Zhao, X.; Urano, K.; Ogasawara, S., Adsorption of polyethylene glycol from aqueous solution on Montmorillonite clays. Colloid Polym. Sci. 1989, 267, 899-906. 
55. Mongondry, P.; Nicolai, T.; Tassin, J. F., Influence of pyrophosphate or polyethylene oxide on the aggregation and gelation of aqueous laponite dispersions. J. Colloid Interface Sci. 2004, 275, 191-196.

56. Nelson, A.; Cosgrove, T., A small-angle neutron scattering study of adsorbed poly(ethylene oxide) on laponite. Langmuir 2004, 20, 2298-2304.

57. Morariu, S.; Bercea, M.; Sacarescu, L., Tailoring of Clay/Poly(ethylene oxide) Hydrogel Properties by Chitosan Incorporation. Ind. Eng. Chem. Res. 2014, 53, 13690-13698. 58. Bassmann, F.; Sequaris, J. M.; Narres, H. D.; Schwuger, M. J., Adsorption of nonionic and anionic polymers on gamma-alumina and Na-montmorillonite and their mixtures. J. Dispersion Sci. Technol. 1999, 20, 607-620.

59. Zaman, A. A.; Tsuchiya, R.; Moudgil, B. M., Adsorption of a low-molecular-weight polyacrylic acid on silica, alumina, and kaolin. J. Colloid Interface Sci. 2002, 256, 73-78.

60. Secor, R. B.; Radke, C. J., Spillover of the diffuse double layer on montmorillonite particles. J. Colloid Interface Sci. 1985, 103, 237-244.

61. Nelson, A.; Cosgrove, T., Small-Angle Neutron Scattering Study of Adsorbed Pluronic Tri-Block Copolymers on Laponite. Langmuir 2005, 21, 9176-9182.

62. Su, C.-C.; Shen, Y.-H., Adsorption of poly(ethylene oxide) on smectite: Effect of layer charge. J. Colloid Interface Sci. 2009, 332, 11-15.

63. Muller, D.; Malmsten, M.; Tanodekaew, S.; Booth, C., Adsorption of diblock copolymers of poly(ethylene oxide) and poly(lactide) at hydrophilic silica from aqueous solution. J. Colloid Interface Sci. 2000, 228, 317-325.

64. Vangeyte, P.; Leyh, B.; Rojas, O. J.; Claesson, P. M.; Heinrich, M.; Auvray, L.; Willet, N.; Jerome, R., Adsorption of poly(ethylene oxide)-b-poly(E-caprolactone) copolymers at the silica-water interface. Langmuir 2005, 21, 2930-2940. 
65. Pavlovic, M.; Adok-Sipiczki, M.; Nardin, C.; Pearson, S.; Bourgeat-Lami, E.; Prevot, V.; Szilagyi, I., Effect of MacroRAFT Copolymer Adsorption on the Colloidal Stability of Layered Double Hydroxide Nanoparticles. Langmuir 2015, 31, 12609-12617.

66. Shen, Y.-H., Estimation of surface area of montmorillonite by ethylene oxide chain adsorption. Chemosphere 2002, 48, 1075-1079.

67. Rossi, S.; Luckham, P. F.; Tadros, T. F., Influence of non-ionic polymers on the rheological behaviour of Na+-montmorillonite clay suspensions. Part II. Homopolymer ethyleneoxide and polypropylene oxide-polyethylene oxide ABA copolymers. Colloids Surf. A: Physicochem. Eng. Asp. 2003, 215, 1-10.

68. Ait-Akbour, R.; Boustingorry, P.; Leroux, F.; Leising, F.; Taviot-Guého, C., Adsorption of PolyCarboxylate Poly(ethylene glycol) (PCP) esters on Montmorillonite (Mmt): Effect of exchangeable cations $(\mathrm{Na}+\mathrm{Mg} 2+$ and $\mathrm{Ca} 2+)$ and PCP molecular structure. J. Colloid Interface Sci. 2015, 437, 227-234.

69. de Souza, C. E. C.; Lima, A. S.; Nascimento, R. S. V., Hydrophobically modified poly(ethylene glycol) as reactive clays inhibitor additive in water-based drilling fluids. $J$. Appl. Polym. Sci. 2010, 117, 857-864.

70. Balazs, A. C.; Siemasko, C. P., Contrasting the surface adsorption of comb and linear polymers. J. Chem. Phys. 1991, 95, 3798-3803.

71. van der Linden, C. C.; Leermakers, F. A. M.; Fleer, G. J., Adsorption of Comb Polymers. Macromolecules 1996, 29, 1000-1005.

72. Striolo, A.; Jayaraman, A.; Genzer, J.; Hall, C. K., Adsorption of comb copolymers on weakly attractive solid surfaces. J. Chem. Phys. 2005, 123, 064710.

73. Theng, B. K. G., Formation and Properties of Clay-Polymer Complexes. Second ed.; Elsevier Science: 2012; Vol. 4, p 511. 
74. van de Wetering, P.; Zuidam, N. J.; van Steenbergen, M. J.; van der Houwen, O. A. G. J.; Underberg, W. J. M.; Hennink, W. E., A Mechanistic Study of the Hydrolytic Stability of Poly(2-(dimethylamino)ethyl methacrylate). Macromolecules 1998, 31, 8063-8068.

75. Cotanda, P.; Wright, D. B.; Tyler, M.; O'Reilly, R. K., A comparative study of the stimuli-responsive properties of DMAEA and DMAEMA containing polymers. J. Polym. Sci. Part A: Polym. Chem. 2013, 51, 3333-3338.

76. Perkins, R.; Brace, R.; Matijević, E., Colloid and surface properties of clay suspensions. I. Laponite CP. J. Colloid Interface Sci. 1974, 48, 417-426.

77. Giles, C. H.; Smith, D.; Huitson, A., General tratment and classification of solute adsorption isotherm.1. Theoretical. J. Colloid Interface Sci. 1974, 47, 755-765.

78. Wu, B.; Li, C.; Yang, H.; Liu, G.; Zhang, G., Formation of Polyelectrolyte Multilayers by Flexible and Semiflexible Chains. J. Phys. Chem. B 2012, 116, 3106-3114.

79. Gregory, J., Rates of flocculation of latex particles by cationic polymers. J. Colloid Interface Sci. 1973, 42, 448-456.

80. Pavlovic, M.; Rouster, P.; Bourgeat-Lami, E.; Prevot, V.; Szilagyi, I., Design of latex-layered double hydroxide composites by tuning the aggregation in suspensions. Soft Matter 2017, 13, 842-851. 